\title{
Delayed decompression exacerbates ischemia-reperfusion injury in cervical compressive myelopathy
}

Pia M. Vidal, ${ }^{1}$ Spyridon K. Karadimas,, ${ }^{1,2}$ Antigona Ulndreaj,, ${ }^{1,2}$ Alex M. Laliberte, ${ }^{1,2}$ Lindsay Tetreault, ${ }^{3}$ Stefania Forner, ${ }^{4}$ Jian Wang, ${ }^{1}$ Warren D. Foltz, ${ }^{5}$ and Michael G. Fehlings ${ }^{1,3}$

'Division of Genetics \& Development, Toronto Western Research Institute and Spine Program, Krembil Neuroscience Center, University Health Network, Toronto, Ontario, Canada. ${ }^{2}$ Institute of Medical Science, ${ }^{3}$ Department of Surgery, Division of Neurosurgery and Spine Program, University of Toronto, Toronto, Ontario, Canada. ${ }^{4}$ Department of Pharmacology, Universidade Federal de Santa Catarina, Florianópolis, Brazil. ${ }^{5}$ Spatio-Temporal Targeting and Amplification of Radiation Responses (STTARR) Innovation Centre, Department of Radiation Oncology, University Health Network, Toronto, Ontario, Canada.

Degenerative cervical myelopathy (DCM) is the most common progressive nontraumatic spinal cord injury. The most common recommended treatment is surgical decompression, although the optimal timing of intervention is an area of ongoing debate. The primary objective of this study was to assess whether a delay in decompression could influence the extent of ischemia-reperfusion injury and alter the trajectory of outcome in DCM. Using a DCM mouse model, we show that decompression acutely led to a 1.5- to 2-fold increase in levels of inflammatory cytokines within the spinal cord. Delayed decompression was associated with exacerbated reperfusion injury, astrogliosis, and poorer neurological recovery. Additionally, delayed decompression was associated with prolonged elevation of inflammatory cytokines and an exacerbated peripheral monocytic inflammatory response $(P<0.01$ and 0.001$)$. In contrast, early decompression led to resolution of reperfusion-mediated inflammation, neurological improvement, and reduced hyperalgesia. Similar findings were observed in subjects from the CSM AOSpine North America and International studies, where delayed decompressive surgery resulted in poorer neurological improvement compared with patients with an earlier intervention. Our data demonstrate that delayed surgical decompression for DCM exacerbates reperfusion injury and is associated with ongoing enhanced levels of cytokine expression, microglia activation, and astrogliosis, and paralleled with poorer neurological recovery.

Conflict of interest: The authors have declared that no conflict of interest exists.

Submitted: December 29, 2016

Accepted: April 27, 2017

Published: June 2, 2017

Reference information: JCI Insight. 2017;2(11):e92512. https:// doi.org/10.1172/jci.insight. 92512

\section{Introduction}

Degenerative cervical myelopathy (DCM) encompasses a spectrum of arthritic and ligamentous conditions of the cervical spine including cervical spondylosis and ossification of the posterior longitudinal ligament (OPLL), which can cause progressive cervical cord compression (1) and result in mild to severe neurologic dysfunction. The spectrum of conditions comprising DCM including cervical spondylotic myelopathy (CSM) and OPLL collectively represent the most common cause of adult spinal cord dysfunction worldwide. Patients with DCM can present with a variety of clinical signs and symptoms, including hand numbness, loss of manual dexterity, and impaired gait pain (2). With increasing life expectancy and the aging of the population, the number of patients affected by DCM is expected to rise; as a result, appropriate treatment strategies must be defined.

Based on recent evidence, surgical decompression is effective at halting disease progression (3) and improving functional status for the majority $(>80 \%)$ of patients $(3,4)$. However, our group has demonstrated that a significant portion of patients $(9.3 \%)$ may experience delayed neurological deterioration and complications. For example, delayed C5 palsy represents the most common complication in the early (within 30 days) postoperative period after decompressive surgery $(3,5,6)$. Furthermore, improvements in functional impairment may vary significantly depending on a number of preoperative factors, including myelopathy severity, duration of symptoms, age, and comorbidities. Specifically, it has been shown that patients are more likely to achieve an excellent score $(\geq 16)$ on the modified Japanese Orthopedic Association scale (mJOA) if they were younger, had a shorter duration of symptoms, and were functionally less severe (7). 


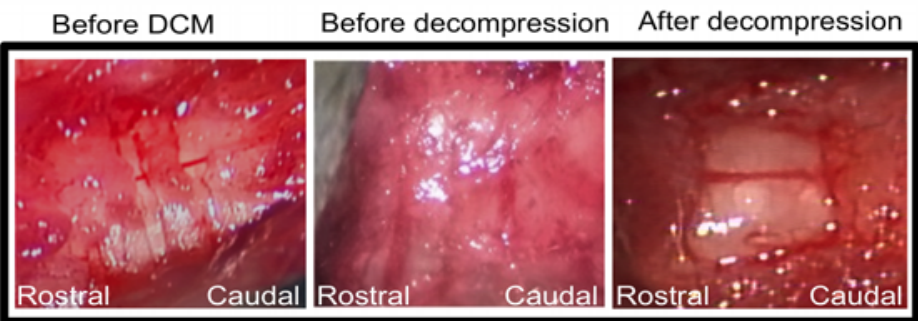

B

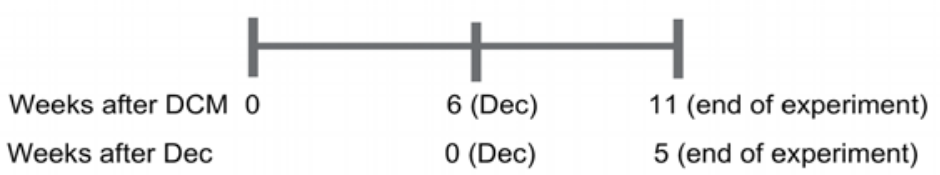

\author{
Early decompression groups \\ DCM-E: Sham Dec
}

DCM-E + Dec: Dec

C

$\begin{array}{lcccc} & & & \text { Delayed decompression groups } \\ \text { Weeks after DCM } & 0 & 12(\mathrm{Dec}) & 17 \text { (end of experiment) } & \text { DCM-D: Sham Dec } \\ \text { Weeks after Dec } & 0(\mathrm{Dec}) & 5 \text { (end of experiment) } & \text { DCM-D + Dec: Dec }\end{array}$

Figure 1. Experimental design. (A) Representative intraoperative images of the spinal cord before material implantation (before DCM), of a compressed animal before and after decompression (from left to right, respectively). (B and C) Scheme of the time points assessed for early and delayed decompression. The time is provided based on (i) weeks after induction of DCM and (ii) after decompression. In these timelines, time 0 refers to either the time of material implantation (see weeks after DCM) or the time of decompression (see weeks after Dec). Mice in the early-decompressed group were operated at 6 weeks after DCM by receiving decompression (DCM-E + Dec) or a sham decompression (DCM-E) (B). Similarly, the delayed-decompressed group received decompression (DCM-D + Dec) or sham decompression (DCM-D), but at 12 weeks after material implantation (C). Both groups were sacrificed at 24 hours and 2 and 5 weeks after their decompressive surgery, and tissues of interest were collected. Neurobehavioral assessment was carried out until 5 weeks after surgery. DCM, degenerative cervical myelopathy; Dec, decompression; DCM-E, age-matched early sham decompressed group; DCM-D, age-matched delayed sham decompressed group.

We recently determined, using a rat model of DCM, that a key factor underlying the postdecompression neurological decline may be explained by ischemia-reperfusion injury (IRI) of the cervical spinal cord (5). However, key knowledge gaps remain regarding the downstream effects of decompression-related IRI, particularly the role of inflammation. Furthermore, it is unclear whether there is a cellular explanation for why duration of symptoms is an important predictor of long-term outcomes in patients undergoing surgery for DCM.

Previous studies have shown that IRI contributes to the injury process in the central nervous system (CNS), through the activation of the immune system (8-12). For example, after ischemic stroke, increased activation of the immune system induces recruitment of immune cells to the affected ischemic parenchyma and triggers the release of inflammatory factors $(9,13)$, thereby increasing the infarct size (9). A similar sequence is observed in the spinal cord and brain following IRI, where rapidly released cytokines (TNF- $\alpha$, CCL-2, CCL-3, CCL-5, CXCL1, IL-1 $\beta$, IL-6) enhance chemotaxis of immune cells $(10,11)$, which in turn perpetuate the inflammatory response and hinder recovery after injury (8). Attenuation of postischemic inflammation within the first 24 hours after cerebral ischemia mitigates secondary injury processes associated with inflammation (14-16). Therapeutic strategies that can resolve these secondary injury processes may allow for improved outcomes in patients treated surgically for DCM.

Herein, we addressed this question using a potentially novel mouse model of DCM, receiving decompression after either a short or long duration of spinal cord compression. Our data demonstrate that restoration of spinal cord blood flow after surgical decompression is seen in parallel with an acute activation of the immune response within the spinal cord. In the early decompression cohort, resolution of inflammation was observed within the first weeks after surgery and was associated with neurological improvement in the upper and lower limbs. However, when surgical decompression was delayed, spinal cord reperfusion 
Table 1. Delay in decompression increases neurological complications in the mouse model of degenerative cervical myelopathy

\begin{tabular}{|c|c|c|c|}
\hline \multicolumn{4}{|c|}{ Motor complications after decompression } \\
\hline Groups & Day 1 & Day 2 & No complications \\
\hline Early decompression (DCM-E + Dec) & 1 animal (4.8\%) & 0 & 20 animals (95.2\%) \\
\hline Delayed decompression (DCM-D + Dec) & 1 animal $(4.5 \%)$ & 3 animals (13.6\%) & 18 animals (81.8\%) \\
\hline
\end{tabular}

was accompanied by a prolonged activation of glial and local immune cells, as well as an increased ratio of peripheral inflammatory/antiinflammatory blood monocytes. Furthermore, delayed surgical decompression led to an increased incidence of neurological complications and absence of neurological recovery in the upper and lower limbs. Consistent with these observations, results from DCM patients with prolonged or shorter duration of symptoms recruited to the CSM AOSpine North America and International studies at 26 centers across the globe provide evidence that the neurological prognosis for DCM patients following surgical decompression is related to the duration of the symptomatic period prior to surgical intervention.

\section{Results}

Delayed surgical decompression for DCM is associated with higher incidence of neurological complications. We designed a randomized blinded experiment using our mouse model of DCM. To mimic the duration of symptoms in humans with DCM, we performed surgical decompression (Dec) on mice with DCM at 2 time points: 1 group underwent early ( 6 weeks after induction of compression, herein referred to as

Mouse model: Early and delayed decompression for Degenerative Cervical Myelopathy

A

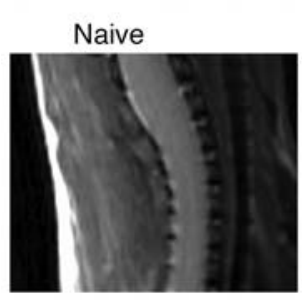

Early decompression group
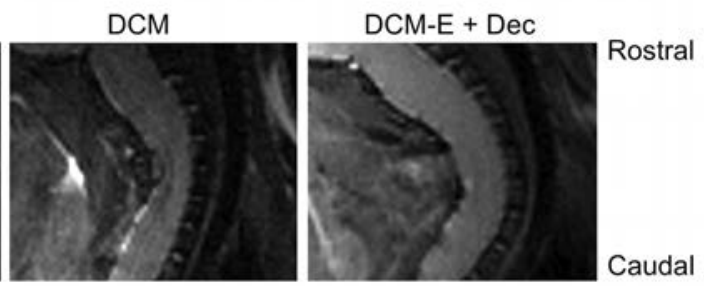

C

Delayed decompression group
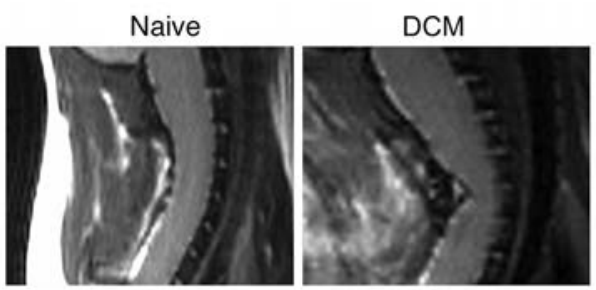

$D C M-D+D e c$

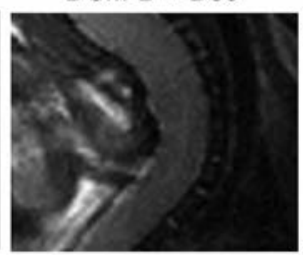

B

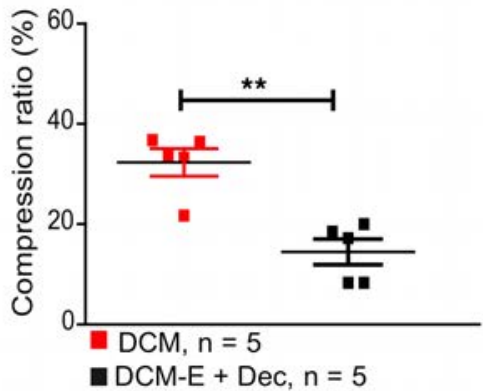

D

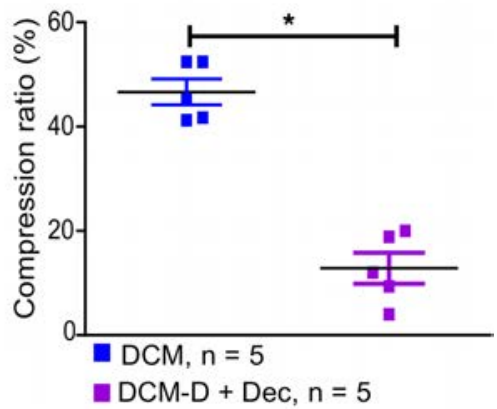

Figure 2. Surgical decompression reduces compression ratio. (A) Representative MR images of mice: naive, 5 weeks after degenerative cervical myelopathy (DCM), and 5 weeks after surgical decompression (DCM-E + Dec). (B) At 1 week before surgical decompression, the DCM group had a 32\% compression ratio, which was significantly reduced to $14.4 \%$ in the DCM-E + Dec group ( $n=5$ animals per group). ${ }^{*} P<0.05$, Mann-Whitney $U$ test. (C) Representative MR images of mice: naive, DCM (at 11 weeks after DCM), and DCM-D + Dec (at 5 weeks after surgical decompression). (D) At 1 week before surgical decompression, the DCM group had a 47\% compression ratio, which was reduced to $12.8 \%$ at 5 weeks after decompression in the DCM-D + Dec group ( $n=5$ per group). ${ }^{*} P<0.05$, Mann-Whitney $U$ test. Compression ratio was calculated based on Fehlings et al. (76), and the data are presented as mean $\pm \mathrm{SEM}$. 
A Spinal cord blood flow technique

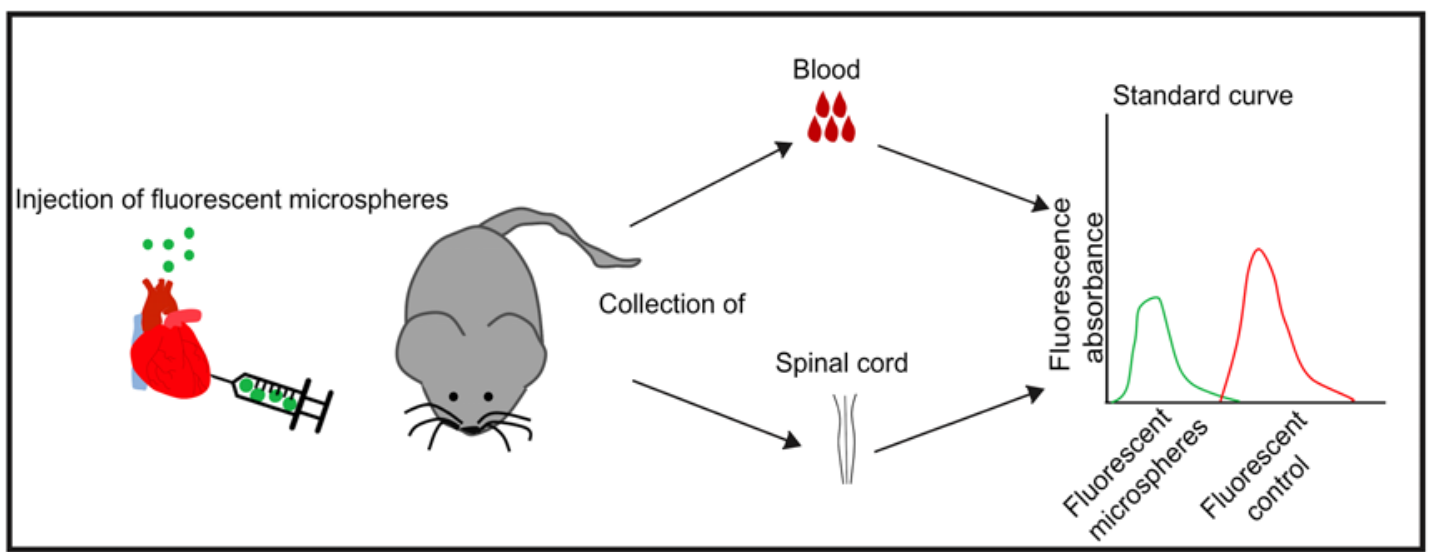

B

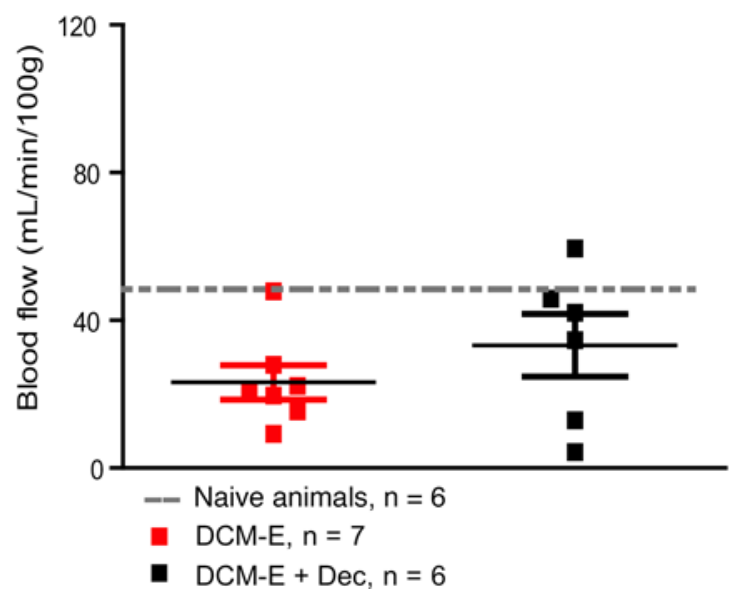

C

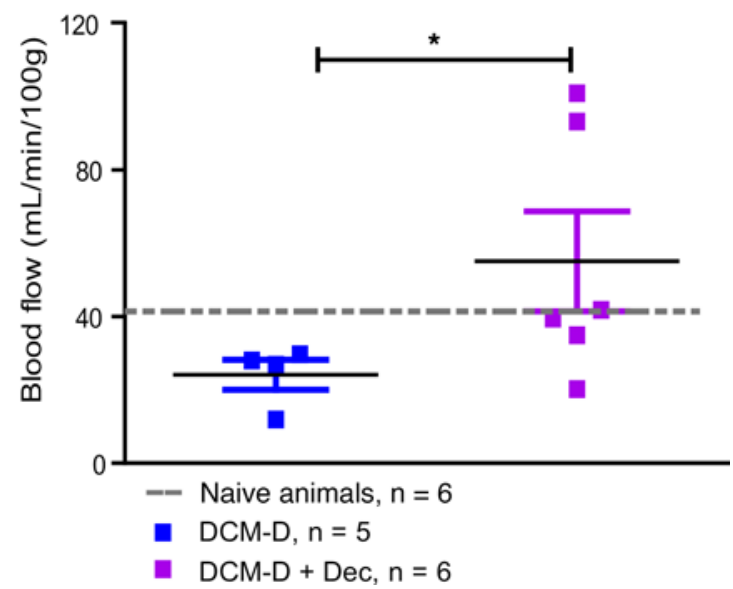

Figure 3. Delayed decompression increases long-term blood flow in the spinal cord. (A) Schematic representation of the technique that measures spinal cord blood flow using fluorescent microparticles injected into the mouse heart. Fluorescence absorbance of microparticles in blood and spinal cord was measured and the obtained values were normalized to a standard curve. (B) At 5 weeks after early decompression, blood flow reached $33.2 \pm 20.8 \mathrm{ml} /$ $\mathrm{min} / 100 \mathrm{~g}$ in the DCM-E + Dec group, whereas animals in the DCM-E group had a blood flow of $23.2 \pm 12.2 \mathrm{ml} / \mathrm{min} / 100 \mathrm{~g}$. Age-matched naive animals had blood flow values of $42.1 \pm 13.5 \mathrm{ml} / \mathrm{min} / 100 \mathrm{~g}$. Naive animals $(n=6)$, DCM-E $(n=7)$, DCM-E + Dec $(n=6)$. (C) At 5 weeks after delayed surgical decompression, blood flow was significantly increased to $55.10 \mathrm{ml} / \mathrm{min} / 100 \mathrm{~g}$ in the DCM-D + Dec group compared with $24.2 \mathrm{ml} / \mathrm{min} / 100 \mathrm{~g}$ in the DCM-D group. ${ }^{*} P$ $<0.05$, Mann-Whitney $U$ test. Age-matched naive animals presented blood flow values of $40 \mathrm{ml} / \mathrm{min} / 100 \mathrm{~g} \pm 13.3 \mathrm{ml} / \mathrm{min} / 100 \mathrm{~g}$. Naive animals $(n=6)$, $\mathrm{DCM}-\mathrm{D}(n=5)$, and DCM-D + Dec $(n=6)$. All data are presented as mean \pm SEM. DCM, degenerative cervical myelopathy; Dec, decompression; DCM-E, agematched early sham decompressed group; DCM-D, age-matched delayed sham decompressed group.

DCM-E + Dec, Figure 1B), and the other group received delayed surgery (12 weeks after induction of compression, herein referred to DCM-D + Dec, Figure 1C). We compared sham-decompressed animals (DCM-E and DCM-D) to age-matched decompressed groups (DCM-E + Dec and DCM-D + Dec, respectively) to avoid cofounding changes in the immune system due to age differences between the groups (17). $\mathrm{T} 1$-weighted $\mathrm{MR}$ images revealed a $32 \% \pm 3 \%$ compression ratio in the DCM-E group (Figure 2, A and B), and a $47 \% \pm 5 \%$ compression ratio in the DCM-D group (Figure 2, C and D) 1 week before surgical decompression (DCM-E versus DCM-D, ${ }^{* *} P<0.01$, Mann-Whitney $U$ test). As expected, compression ratios were significantly reduced at 5 weeks after surgical decompression in both groups compared with their corresponding DCM group before surgical decompression (Figure 2, B and D). In line with our previous study (5), we observed postoperative neurological complications in our animals following decompression. Specifically, at 24 hours after surgery, $4.8 \%$ ( 1 out of 21 ) of the animals receiving early decompression presented gross motor complications (defined as reduced ankle movement and plantar stepping, upper and/or lower limb stiffness and/or weakness, and forepaw palsy), compared with $13.6 \%$ (3 out of 22) of animals who underwent delayed decompression (Table 1). At 48 hours after decompression, while no complications 
Cytokine production after decompression
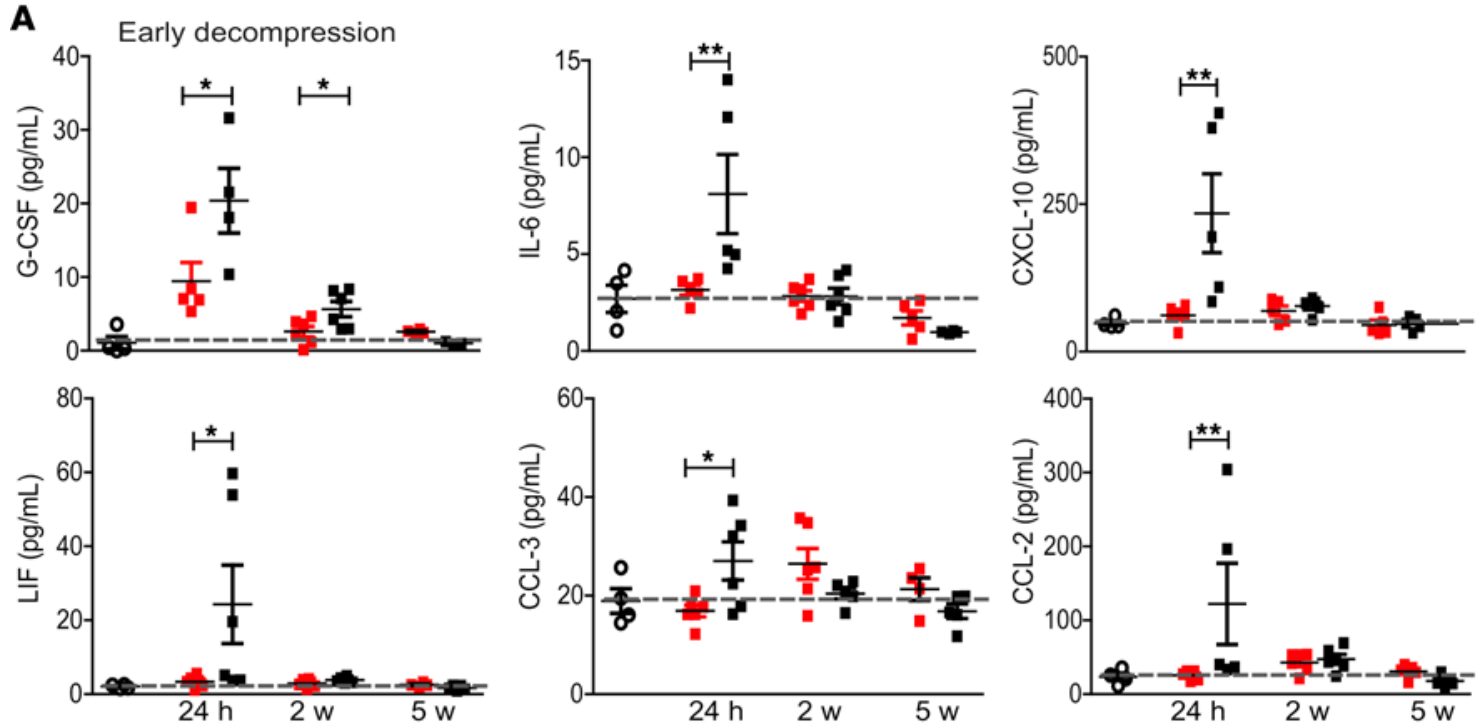

-o Naive animals, $n=3-4$

B
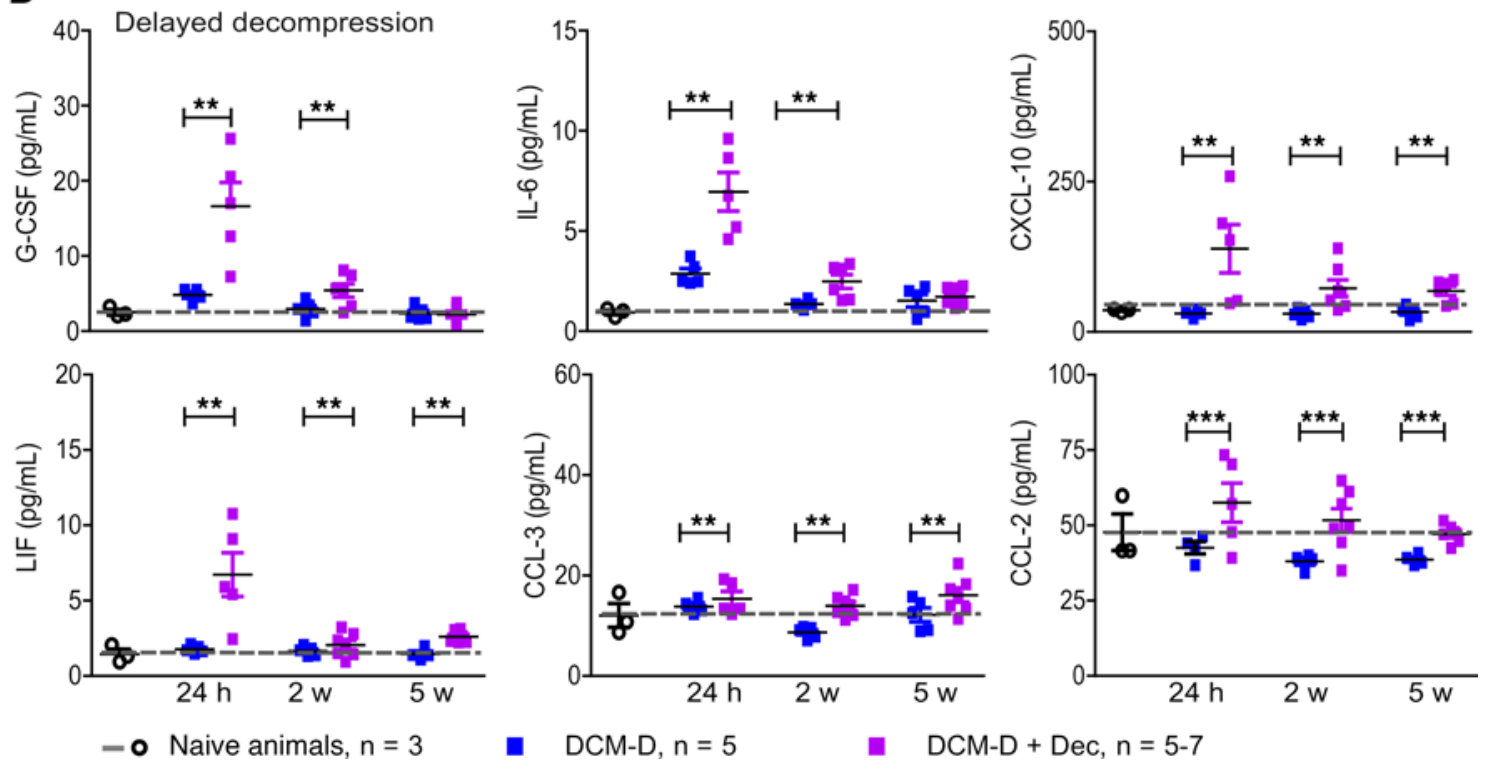

Figure 4. Surgical decompression triggers acute release of cytokines within the spinal cord, which persist after delayed decompression. (A) ELISA results from homogenized spinal cord tissue indicated increased levels of inflammatory factors (G-CSF, IL-6, CXCL10, LIF, CCL-3, CCL-2) in the DCM-E + Dec group compared with the DCM-E group acutely after decompression (at 24 hours). ${ }^{*} P<0.05$; ${ }^{*} P<0.01$, two-way ANOVA, Bonferroni post-hoc. A group of naive animals with the same age as the animals at 5 weeks after decompression was also included. Naive $(n=3-4)$, DCM-E $(n=5)$, and DCM-E + DC groups $(n=5-6)$. (B) At 24 hours after delayed decompression, the DCM-D + Dec group presented a significant increase levels of G-CSF, IL-6, CXCL10, LIF, CCL-3, and CCL-2 compared with the DCM-D group. Moreover, levels of CXCL10, LIF, CCL-3, and CCL-2 were elevated at 2 and 5 weeks in the DCM-D + Dec group compared with the DCM-D group. Naive animals matching the age of animals at 5 weeks after decompression were included for reference. ${ }^{*} P<0.05$; ${ }^{* *} P<0.01 ;{ }^{* *} P \leq 0.001$, two-way ANOVA, Bonferroni post-hoc. Naive $(n=3), \mathrm{DCM}-\mathrm{D}(n=5), \mathrm{DCM}-\mathrm{D}+\mathrm{Dec}(n=5-7)$. Data are presented as mean \pm SEM. DCM, degenerative cervical myelopathy; Dec, decompression; DCM-E, age-matched early sham decompressed group; DCM-D, age-matched delayed sham decompressed group; h, hours; w, weeks.

presented in the group that underwent early decompression, 4.5\% (1 out of 22) of animals that received delayed decompression developed complications (Table 1).

Prolonged spinal cord ischemia increases spinal cord blood flow after surgical decompression. Our previous study demonstrated that surgical decompression increases spinal cord blood flow and induces a reperfusion injury as early as 24 hours following decompression (5). However, whether the duration of spinal cord compression prior to decompression influences the extent of reperfusion injury over time has yet to be examined. 
Increased ratio of inflammatory/patrolling blood monocytes after delayed decompression

A 2 weeks: Early decompression
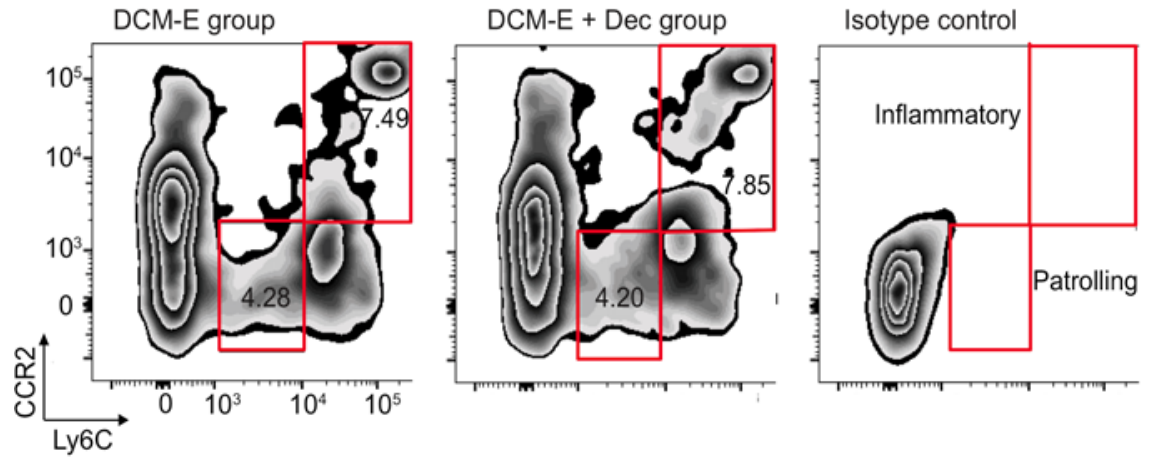

C

2 weeks: Delayed decompression

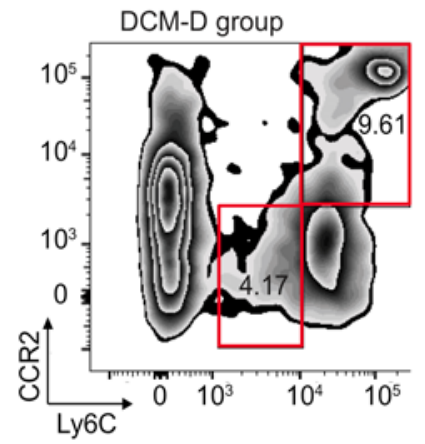

B

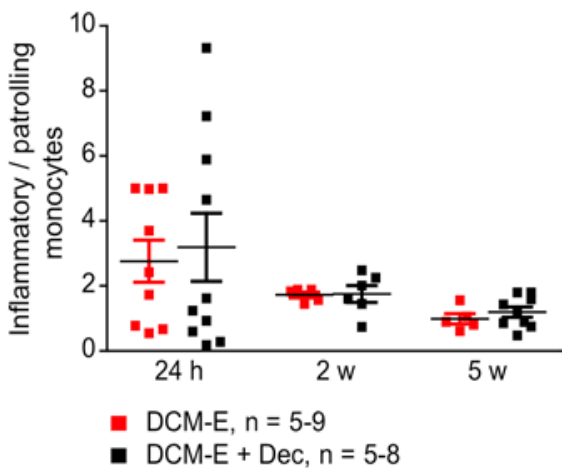

D
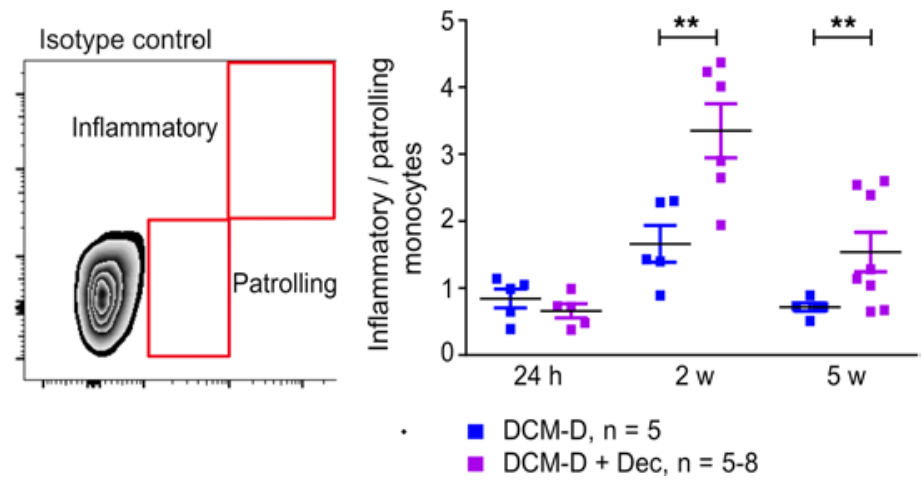

Figure 5. Delayed surgical decompression increases the ratio of inflammatory/patrolling blood monocytes. (A) Blood samples were transcardially collected at selected time points in the DCM-E and DCM-E + Dec groups. Representative contour plots of blood monocytes for DCM-E, DCM-E + Dec, and isotype controls at 2 weeks after surgical decompression. Inflammatory monocytes were gated as Ly6ChiCCR2 ${ }^{+}$(upper red panel) and patrolling monocytes as Ly6C ${ }^{\circ} \mathrm{CCR2}$ - (lower red panel). (B) The ratio of inflammatory/patrolling monocytes was not significantly different between the DCM-E and DCM-E + Dec groups at all time points (24 hours [DCM-E, $n=9$; DCM-E + Dec, $n=10$ ], 2 weeks [DCM-E, $n=5$; DCM-E + Dec, $n=6$ ], and 5 weeks [DCM-E, $n=5$; DCM-E + Dec, $n=9]$ ). (C) Representative contour plots of inflammatory and patrolling blood monocytes for DCM-D, DCM-D + Dec, and isotype control at 2 weeks after surgical decompression. (D) The ratio of inflammatory/patrolling monocytes was similar between DCM-D and DCM-D + Dec groups at 24 hours after surgery (DCM-D, $n=5 ; D C M-D+D e c, n=5$ ). However, this ratio was higher in the group receiving delayed decompression as compared with the same group, at 2 and 5 weeks after decompression (DCM-D, $n=5 ; D C M-D+D e c, n=5-8)$. ${ }^{* *} P<0.01$, two-way ANOVA. Data are presented as mean \pm SEM. DCM, degenerative cervical myelopathy; Dec, decompression; DCM-E, age-matched early sham decompressed group; DCM-D, age-matched delayed sham decompressed group.

To start addressing this issue, we measured the spinal cord blood flow by injecting fluorescent microspheres into the mouse heart, as shown in the diagram in Figure 3A, at 5 weeks after decompressive surgery. We observed a 50\% reduction in blood flow in both DCM-E and DCM-D groups compared with age-matched naive animals (Figure 3, B and C). No significant differences were observed between DCM-E and DCM-D groups. Compared with DCM-E, the DCM-E + Dec group had increased blood flow by $34.6 \%$ (Figure 3B), although this did not reach statistical significance. Blood flow in the DCM-D + Dec group was significantly increased compared with the DCM-D group by $43.8 \%$ (Figure 3C).

Delayed surgical decompression induces a sustained systemic and local postischemic inflammatory response. Excessive activation of the immune system has been reported during reperfusion (18). To assess whether the reperfusion induces an inflammatory response, we measured the spinal cord levels of key cytokines with ELISA at 3 relevant time points. We compared decompressed mice with age-matched sham-decompressed animals to avoid any changes in the immune response associated with immunosenescence rather than due to decompression (P.M. Vidal, unpublished observations and refs. 17, 19). Surgical decompression caused significant increase in the production of G-CSF, IL-6, CXCL10, LIF, CCL-3, and CCL-2 at 24 hours after surgical decompression, as compared with the age-matched DCM group (Figure 4). In the DCM-E + Dec and DCM-D + Dec groups, the levels of G-CSF remained 
A Surgical decompression does not affect the number of microglia cells
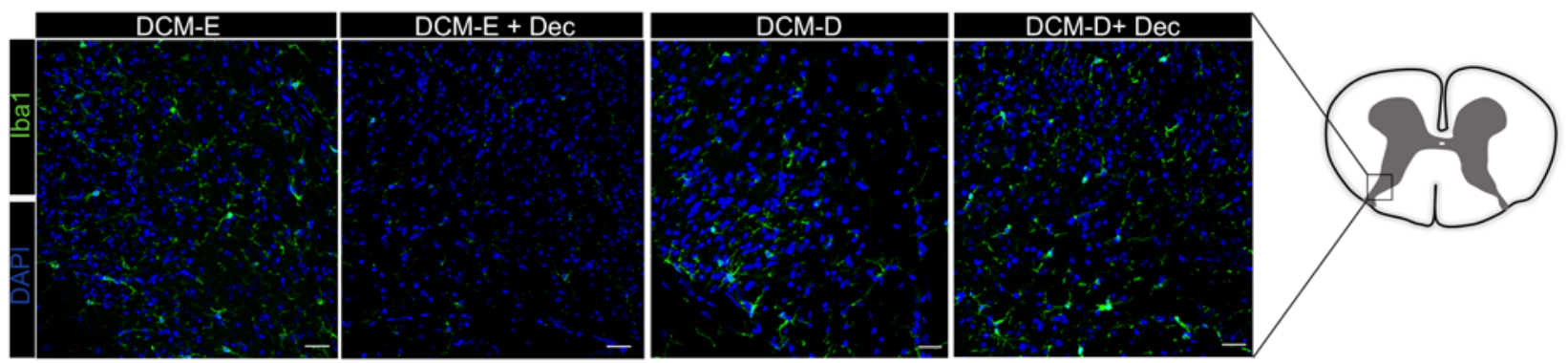

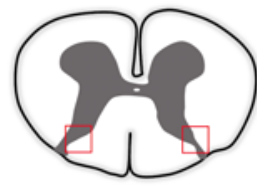

B

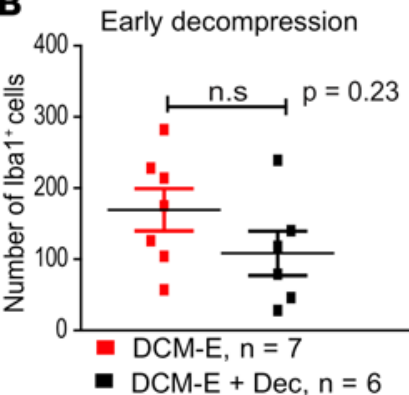

E

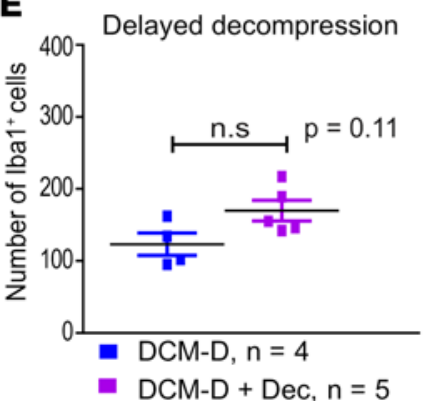

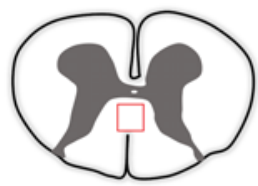

C

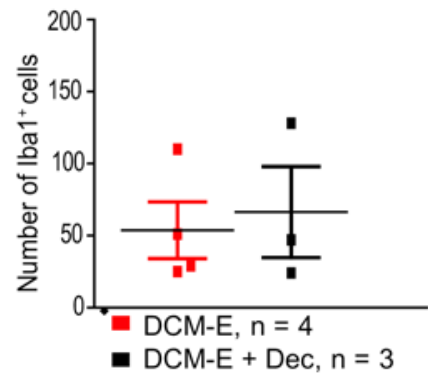

$\mathbf{F}$

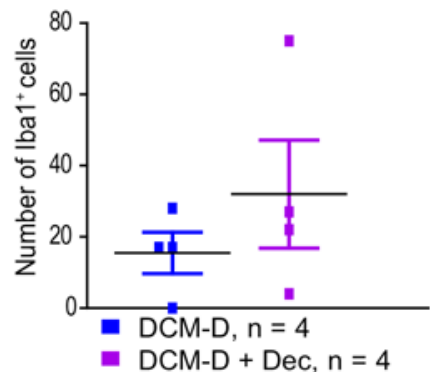

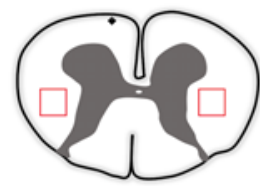

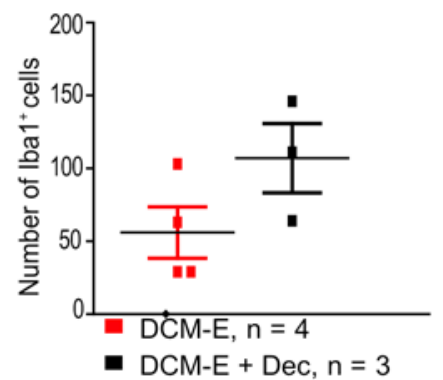

G

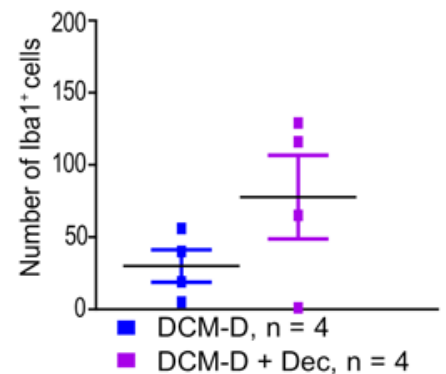

Figure 6. Surgical decompression does not affect the number of recruited microglia/macrophages in the spinal cord. (A) Representative images of Iba1 ${ }^{+}$ cells in the dorsal horn from mice at 5 weeks after either early or delayed decompression and age-matched sham controls. (B) At 5 weeks after decompression, Iba1 $1^{+}$cells were quantified in the regions of dorsal horns outlined by red squares, as indicated in the spinal cord diagram. A slight decrease in Iba1 ${ }^{+}$ cells was detected in the dorsal horns of the DCM-E + Dec group $(n=6)$ compared with the DCM-E group $(n=7)$. (C and $\mathbf{D})$ No significant differences were observed in the DCM-E group $(n=4)$ compared with the DCM-E + Dec $(n=3)$ in the dorsal columns (C) and the lateral corticospinal tracts (D). In all cases, the analyzed area is represented by the red squares in the diagram of the spinal cord. (E-G) No significant changes were observed in the number of Iba1 ${ }^{+}$ cells in the dorsal horns $(\mathbf{E})$, dorsal columns $(\mathbf{F})$, and lateral corticospinal tracts $(\mathbf{C})$ between the DCM-D $(n=4)$ and DCM-D + Dec $(n=5-4)$ at 5 weeks after decompression. All the results are presented as mean \pm SEM of 5 to 6 slides per animal. Scale bars: $25 \mu \mathrm{m}$. DCM, degenerative cervical myelopathy; Dec, decompression; DCM-E, age-matched early sham decompressed group; DCM-D, age-matched delayed sham decompressed group.

elevated for 2 weeks after surgery, but these differences were no longer present at 5 weeks after surgery. In contrast, in the DCM-D + Dec group, IL-6 remained elevated for 2 weeks after surgical decompression (Figure 4B). Furthermore, we observed a prolonged increase in LIF, CCL-3, CCL-2, and CXCL10 (Figure 4B) that lasted for 5 weeks after surgery in the DCM-D + Dec group compared with the DCMD group. Of note, significant changes were observed between DCM-E and DCM-D in the production of CXCL10, CCL-2 $(P<0.01$, two-tailed $t$ test), and LIF $(P<0.05$, two-tailed $t$ test) at 24 hours after sham decompression.

Changes in peripheral immune cell populations, especially in blood monocytes, have been shown to occur after ischemic stroke and to correlate with clinical recovery after hip surgery $(20,21)$. Blood monocytes give rise to tissue macrophages and dendritic cells, and have been shown to be good predictors of tissue injury severity in certain pathologies (22). Based on the sustained inflammatory state in the spinal cord following 
Delayed decompression increases the expression of Galectin-3

\section{A Early decompression}
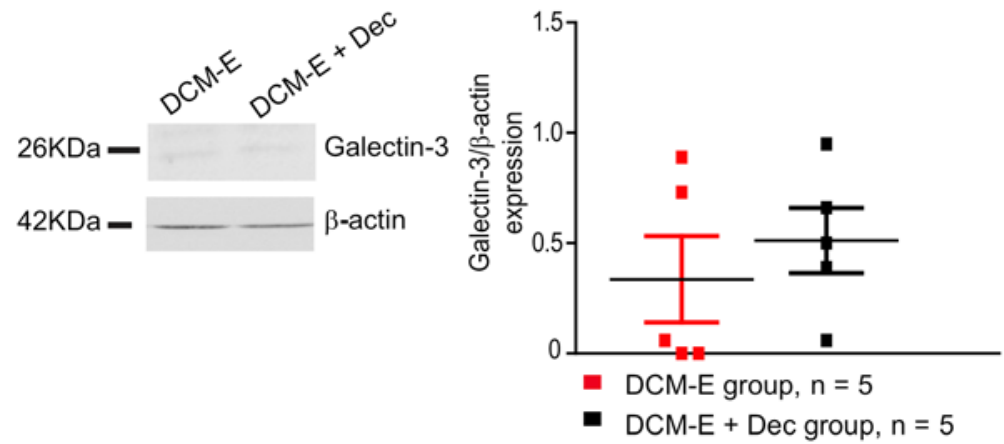

B Delayed decompression

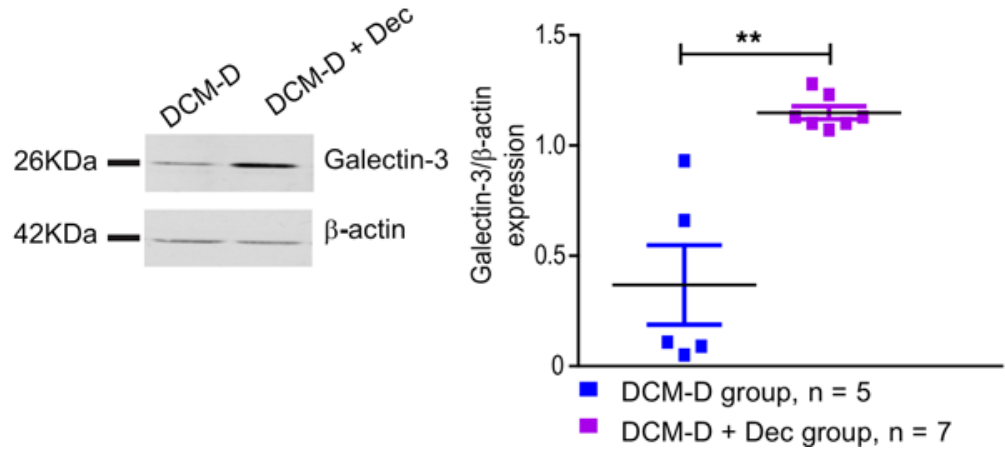

Figure 7. Delayed decompression induces increased levels of Galectin-3 in the spinal cord. (A) Representative Western blot results of Galectin-3 expression and their respective loading control $\beta$-actin for the DCM-E $(n=5)$ and DCM-E + Dec $(n=5)$ groups at 5 weeks after decompression. Densitometric quantification revealed minor expression of Galectin-3 in the spinal cord from both groups. Data were analyzed using a Mann-Whitney $U$ test. (B) Representative Western blot of Galectin-3 expression and $\beta$-actin in the DCM-D and DCM-D + Dec groups at 5 weeks after decompression. Quantification of Galectin-3 in DCM-D $(n=5)$ and DCM-D + Dec $(n=7)$ groups shows a significant increase in the expression of this marker at 5 weeks after decompression. ${ }^{* *} P<0.01$, Mann-Whitney $U$ test. DCM, degenerative cervical myelopathy; Dec, decompression; DCM-E, agematched early sham decompressed group; DCM-D, age-matched delayed sham decompressed group.

delayed decompression, we hypothesized that blood monocytes will display an inflammatory state after surgery. We focused on the changes in the ratio of inflammatory to patrolling monocytes (Ly6C ${ }^{\text {hi }} \mathrm{CCR} 22^{+} / \mathrm{Ly} 6 \mathrm{C}^{\text {lo }} \mathrm{CCR} 2^{-}$) at different time points during compression and after decompression. An increased ratio between these cells has been shown to correlate with damage following postischemic events in the brain (21). We did not observe significant changes in the ratio of circulating inflammatory/patrolling monocytes

between DCM-E and DCM-E + Dec (Figure 5, A and B). However, there was a 1.5-fold increase in this ratio at 2 weeks after decompression in the DCM-D + Dec group (Figure 5, C and D). The increase in this ratio was also present at 5 weeks after delayed decompression, although at a lower magnitude (Figure 5, C and D). Of note, a significant decrease in the ratio of circulating inflammatory/patrolling monocytes was observed between DCM-E and DCM-D at 24 hours after sham surgery $(P<0.05$, two-tailed $t$ test).

Early decompression reduces astrogliosis in contrast to delay decompression. Microglia/macrophages and astrocytes play key roles during postischemic inflammation, by producing inflammatory mediators and reactive oxygen species (ROS) $(15,23-25)$. Activation and recruitment of these cells has been shown to be prominent in human and experimental models of $\operatorname{DCM}(5,26-28)$. In order to determine if surgical decompression has an effect on their recruitment, we performed immunohistochemistry on cervical spinal cord sections for the markers ionized calcium-binding adapter molecule 1 (Iba1) and glial fibrillary acidic protein (GFAP) at 5 weeks after surgical decompression. Iba ${ }^{+}$cells were located in the white and gray matter, as well as around the central canal of DCM of decompressed mice, as shown in the spinal cord diagrams (Figure 6A). Based on previous studies in DCM (28), our analysis was focused on the number of Iba1 ${ }^{+}$cells in the dorsal horn, dorsal columns, and around the lateral corticospinal tracts (Figure 6). Contrary to our hypothesis, surgical decompression did not significantly alter the number of $\mathrm{Iba}^{+}$cells after surgical decompression (Figure 6, B-G). However, when we assessed the expression of Galectin-3, which is considered a regulator of activated/proliferating microglia/macrophages after cerebral ischemia-reperfusion (29) and reactive astrocytes (30), using Western blotting we observed low expression in the DCM-E and DCM-E + Dec groups (Figure 7A), but strong expression in the DCM-D + Dec group compared with the DCM-D group (Figure 7B; see complete unedited blots in the supplemental material available online with this article; https://doi. org/10.1172/jci.insight.92512DS1).

We next measured the extent of astrogliosis in the dorsal horn, the area of the spinal cord where we observed the highest numbers of $\mathrm{Iba}^{+}$cells following decompression. GFAP immunoreactivity was quantified in spinal cord samples around the C5-C6 level of compressed and surgically decompressed animals at 5 weeks after surgical decompression (Figure 8A). Quantification of GFAP immunoreactivity over a 
A Delayed decompression promotes spinal cord astrogliosis
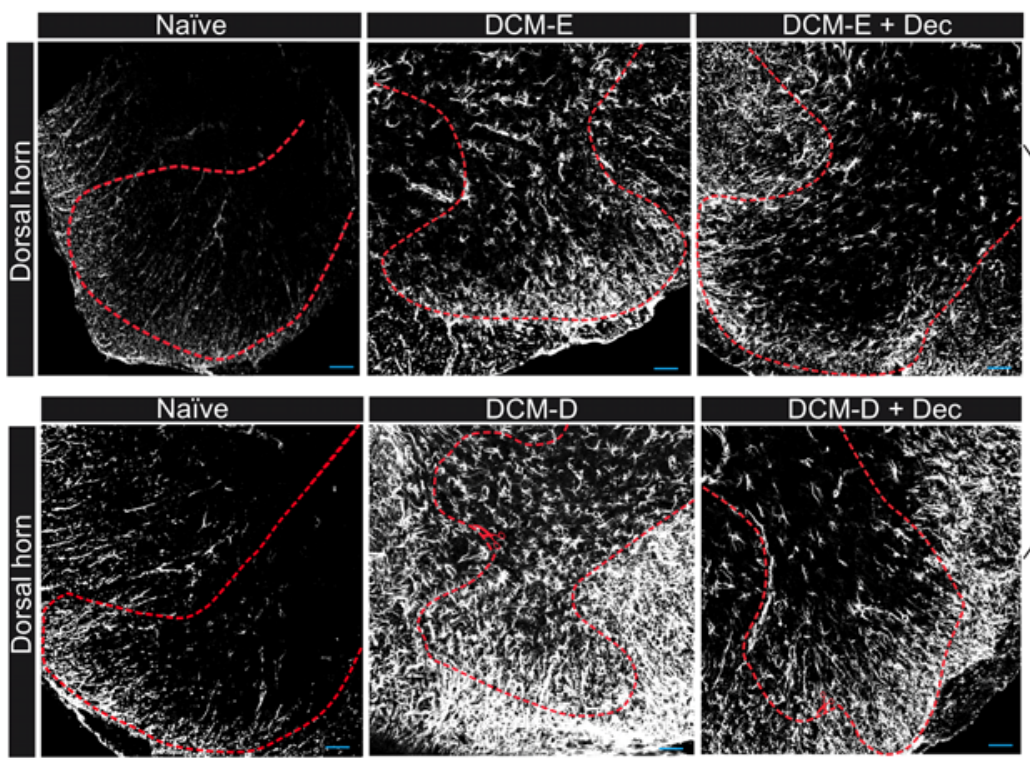

B

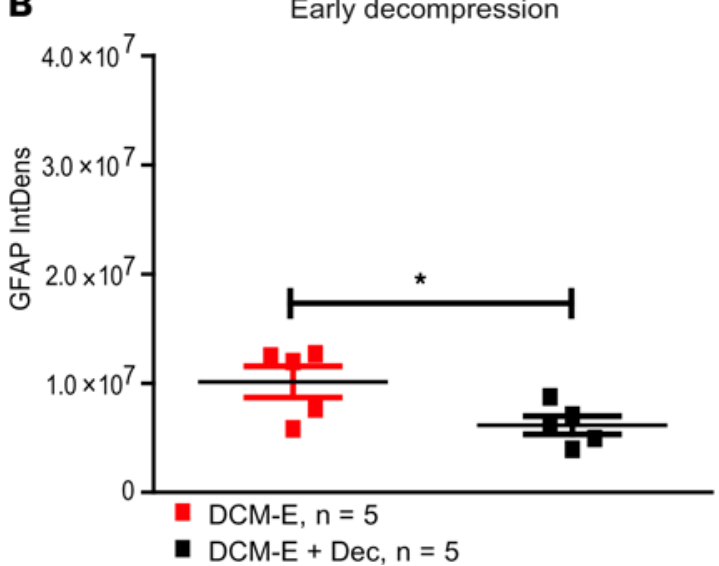

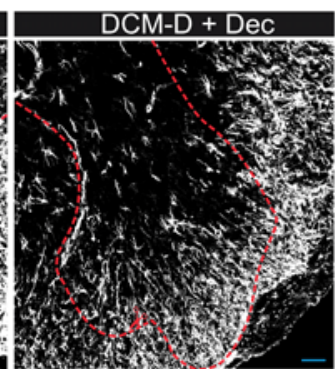

C

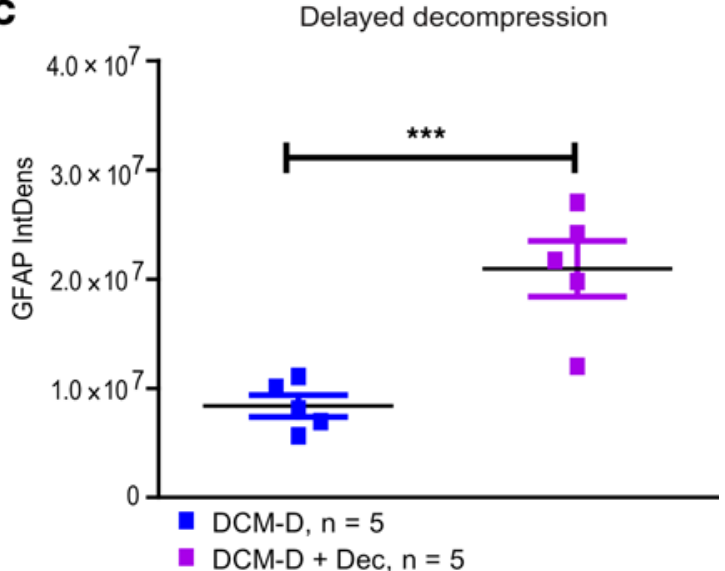

Figure 8. Early decompression attenuates astrogliosis in the dorsal horns. (A) Representative confocal images of dorsal horns from mice that underwent early or delayed decompression, their age-matched sham controls, and age-matched naive mice, all stained for glial fibrillary acidic protein (GFAP). The red dotted lines delineate the dorsal horns where GFAP immunoreactivity was quantified. The spinal cord diagram on the right side represents the area of constant size within the dorsal horns, used for the analysis of GFAP immunoreactivity. (B) GFAP immunoreactivity was significantly reduced at 5 weeks after decompression in the DCM-E + Dec $(n=5)$ group compared with the age-matched DCM-E group $(n=5) .{ }^{*} P<0.05$, Mann-Whitney $U$ test. (C) Astrogliosis was significantly increased in the dorsal horns of DCM-D + Dec $(n=5)$ compared with the DCM-D group $(n=5)$. ${ }^{* * *} P<0.001$, Mann-Whitney $U$ test. All the results are presented as mean \pm SEM. Scale bars: $25 \mu \mathrm{m}$. DCM, degenerative cervical myelopathy; Dec, decompression; DCM-E, age-matched early sham decompressed group; DCM-D, age-matched delayed sham decompressed group; IntDens, integrated density.

constant area revealed a reduction in dorsal horn astrogliosis in the DCM-E + Dec group (Figure 8B) but increased astrogliosis in the DCM-D + Dec group (Figure 8C) at 5 weeks after decompression.

Early decompression leads to gait improvement in the upper and lower extremities. To investigate whether the changes observed after surgical decompression in the spinal cord are associated with functional outcomes, we performed locomotion analysis in DCM and decompressed animals. Gait impairment is considered to be one of the first symptoms of DCM in patients (31); in particular, swing speed and stride length are 2 gait parameters known to be affected by progressive spinal cord compression (32). We used the CatWalk system to measure gait deficits before and after decompression without significant changes in speed between the compared groups (33). Representative footprints from mice with DCM before and after undergoing early or delayed decompression using CatWalk are shown in Figure 9, A and B. Since the speed of locomotion is more similar within DCM-E and DCM-E + Dec or DCM-D and DCM-D + Dec groups, and that the speed of locomotion is correlated to many gait parameters, we examined the relative effects of decompression independently at each time point. 
Early surgical decompression attenuates locomotor deficits

A Early decompression

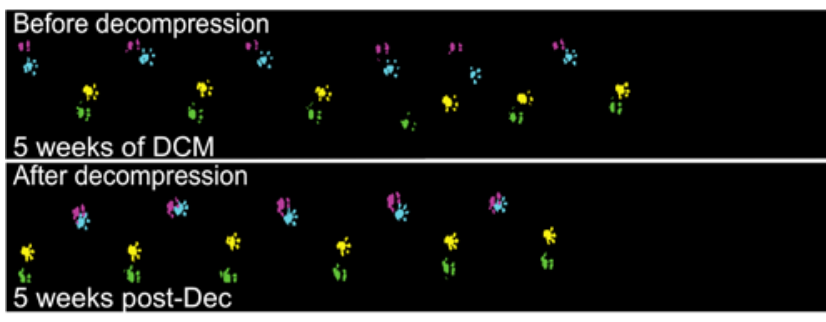

B Delayed decompression

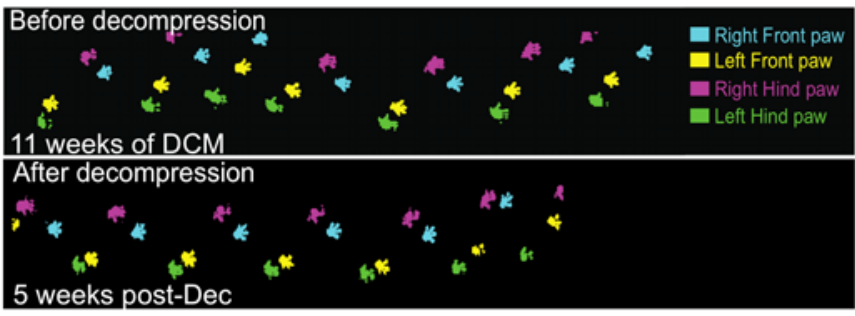

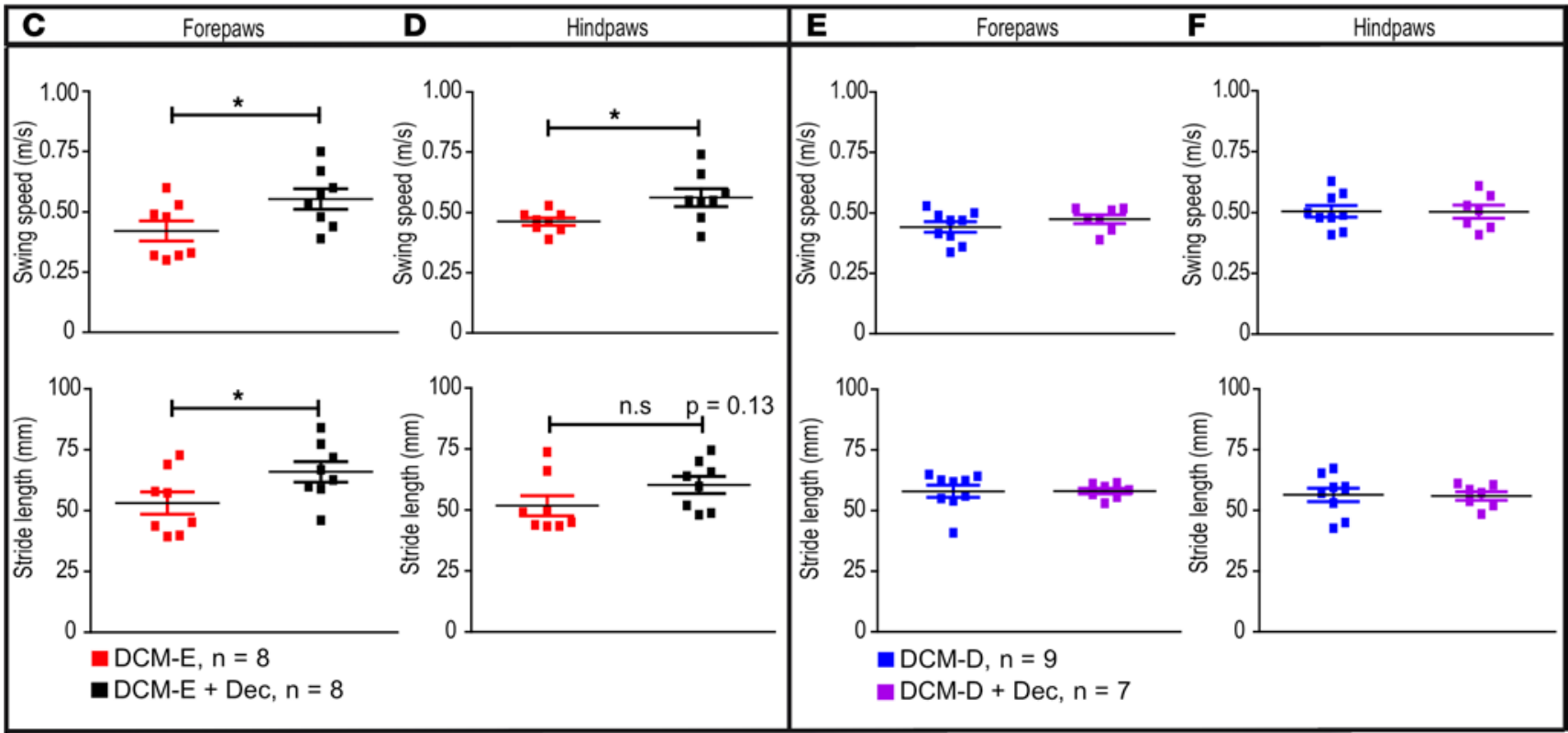

Figure 9. Early surgical decompression attenuates gait deficits. (A and B) Representative footprints from mice with DCM before and after undergoing early (A) or delayed (B) decompression using CatWalk. (C) In the forepaws, swing speed and stride length increased significantly at 5 weeks in the DCM-E + Dec group compared with the DCM-E group. ${ }^{*} P<0.05$, Mann-Whitney $U$ test. (D) In hind limbs, only swing speed was significantly increased $(n=8$ animals per group, data represent the mean of 3 runs per animal). ${ }^{*} P<0.05$, Mann-Whitney $U$ test. (E and $\mathbf{F}$ ) Delayed decompression did not attenuate gait deficits in any of the limbs in the DCM-D + Dec group $(n=7)$ compared with the DCM-D group $(n=9)$ at 5 weeks after surgery. Mann-Whitney $U$ test. All data are presented as mean \pm SEM. DCM, degenerative cervical myelopathy; Dec, decompression; DCM-E, age-matched early sham decompressed group $\mathrm{DCM}-\mathrm{D}$, age-matched delayed sham decompressed group.

During the first 2 weeks after surgical decompression, we did not observe any significant changes in swing speed and stride length between groups (data not shown). However, at 5 weeks after early decompression, we observed a 1.5-fold improvement in swing speed in forepaws (Figure 9C) and a 1.2fold increase in hind paws (Figure 9D) of animals in the DCM-E + Dec group, compared with DCM-E. Stride length also recovered after decompression in this group (Figure 9, C and D), but only reached statistical significance in the forepaws (Figure 9C). In contrast, no significant recovery of swing speed or stride length was found in the group receiving a delayed decompression (DCM-D + Dec) compared with the DCM-D group (Figure 9, E and F).

Interlimb coordination was assessed using the CatWalk system (step pattern) and rotarod (data not shown) in naive, compressed, and decompressed animals. With the CatWalk system, we focused on the 3 main categories of normal step sequence pattern in rodents: cruciate, alternate, and rotary (34). The alternate pattern is the most frequently observed in rodents $(35,36)$. In naive animals we observed alternate $(80 \%)$ and cruciate $(20 \%)$ stepping patterns (Supplemental Figure 1A). The step pattern and the speed of our animals during DCM and after surgical decompression were visibly affected. During DCM the alternate pattern was decreased to $33.1 \%$ in the DCM-E group and to 59.2\% in the DCM-D group (Supplemental Figure 1, B and D). Early decompression led to an $18 \%$ improvement in the alternate step sequence pattern (in which left front paw is followed by 
Table 2. Demographic baseline characteristics and clinical data for degenerative cervical myelopathy patients

\begin{tabular}{|c|c|}
\hline \multicolumn{2}{|l|}{ Patients demographic information } \\
\hline \multicolumn{2}{|l|}{ Gender: } \\
\hline Women & 213 \\
\hline Men & 337 \\
\hline Mean age (years) & $57.9 \pm 12.08$ \\
\hline \multirow[t]{2}{*}{ Smoking status } & 151 smokers \\
\hline & 399 nonsmokers \\
\hline Mean number of decompressed segments & $3.9 \pm 1.3$ (2-7 levels) \\
\hline Average duration of surgery & $184.8 \pm 83.07$ minutes \\
\hline \multicolumn{2}{|l|}{ Type of surgery: } \\
\hline Anterior & 293 \\
\hline Posterior & 238 \\
\hline Combined & 19 \\
\hline
\end{tabular}

right hind paw), compared with 3\% improvement in the delayed group (Supplemental Figure 1). The alternate pattern is more frequent during slow to moderate speeds, whereas the cruciate pattern is associated with gallop or trot (37). So while it may appear that delayed DCM groups are closer to sham in step pattern, this interpretation is limited due to other concurrent changes in the DCM gait phenotype, specifically speed. Indeed, the fact that DCM-D + Dec was slower than the DCM-E + Dec group (data not shown) suggests that these animals employed the alternate pattern more frequently due to this reduced speed. Additionally, some animals presented a rotary step pattern after early and delayed decompression (Supplemental Figure 1).

Early decompression improves manual dexterity. Neurological deficits in the upper extremities are considered the second most frequent and debilitating symptom of DCM in patients (38). For this reason, we characterized the upper limb deficits of mice with DCM before and after surgical decompression, using the wire hang test (39) and the Capellini handling test (40). When we assessed grip/muscle strength with the wire hang test (Figure 10C), we found that the DCM-E + Dec group exhibited an increased latency to fall compared with DCM-E animals, at all time points (Figure 10A). In contrast, DCM-D + Dec did not show significant improvement compared with the DCM-D group (Figure 10B). Manual dexterity is often markedly affected during chronic spinal cord compression. Therefore, we examined whether decompression would improve manual dexterity by using the Capellini handling test (Figure 10F) (40). DCM-E and DCM-D groups showed a 2-fold increase in the time spent eating a piece of pasta compared with age-matched naive animals (Figure $10, \mathrm{D}$ and $\mathrm{E})$. In agreement with the wire hang test, the DCM-E + Dec group showed significantly reduced eating time at 3 to 4 days after surgery. This improvement in manual dexterity was sustained for up to 5 weeks after surgery (Figure 10D). However, animals in the DCM-D + Dec group did not show improved manual dexterity compared with the DCM-D group (Figure 10E).

Hyperalgesia is reduced by early surgical decompression. The development of pain is another symptom observed in a proportion of DCM patients (41) and in animal models of DCM (32). To assess the development of pain sensitivity during DCM and after decompressive surgery, we measured mechanical hyperalgesia in the paws by measuring the frequency of response to a von Frey filament before DCM induction (baseline, Bsl), 1 week before decompression, and at 1, 2, and 5 weeks after surgical decompression. Significant differences between Bsl and 1 week before decompression were observed in the forepaws of DCM-E and DCM-D groups (Figure 11, A and C). Animals with DCM start to progressively develop upper extremity hypersensitivity at 3 weeks after induction of DCM (data not shown), reaching a plateau response at the week of early decompression (Figure 11A, week 0). One week before decompression, animals in the DCM-E and DCM-D groups retracted their forepaws to the stimulus with a frequency of $34 \%$ to $40.8 \%$ (Figure 11, A and C). Surgical decompression in the DCM-E + Dec group significantly attenuated pain response from week 1 until week 5 after early decompression (Figure 11A). However, no effect on pain response was observed in the DCM-D + Dec group compared with the DCM-D group in the forepaws (Figure 11C). Surgical decompression did not significantly affect pain response in the hind paw of the DCM-E or DCM-D group (Figure 11, B and D).

Table 3. Differences in functional outcomes between patients with a short or long duration of symptoms from the AOSpine North American and International studies

\begin{tabular}{|c|c|c|c|}
\hline & $\begin{array}{l}\text { Patients with a duration } \\
\text { of symptoms }<6 \text { months }\end{array}$ & $\begin{array}{l}\text { Patients with a duration } \\
\text { of symptoms } \geq 6 \text { months }\end{array}$ & $P$ value \\
\hline$\triangle \mathrm{mJOA}$ (from baseline to 24 months) ${ }^{\mathrm{A}}$ & 4.11 (95\% Cl: 3.63 to 4.58$)$ & 3.15 (95\% Cl: 2.88 to 3.41$)$ & 0.0006 \\
\hline$\Delta \mathrm{mJOA}$ (from baseline to 24 months) ${ }^{\mathrm{B}}$ & 4.00 (95\% Cl: 3.54 to 4.47$)$ & 3.18 (95\% Cl: 2.92 to 3.44$)$ & 0.0027 \\
\hline Proportion achieving an MCID on the mJOA & $82.2 \%$ & $67.1 \%$ & $0.0016^{\mathrm{c}}$ \\
\hline
\end{tabular}


Early surgical decompression improves upper extremity function

A

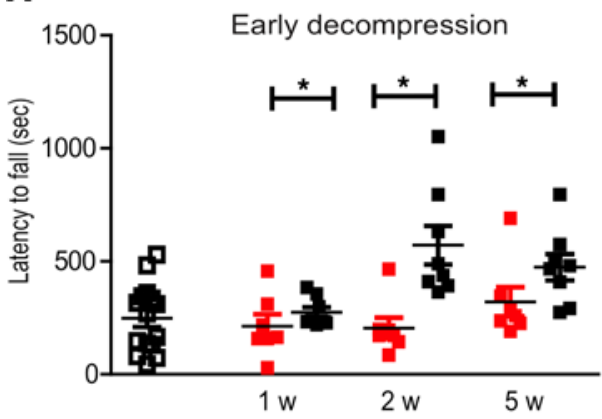

$\square \mathrm{DCM}$ before surgical decompression, $\mathrm{n}=15$ DCM-E, $\mathrm{n}=7$

-DCM-E + Dec, $\mathrm{n}=8$

B

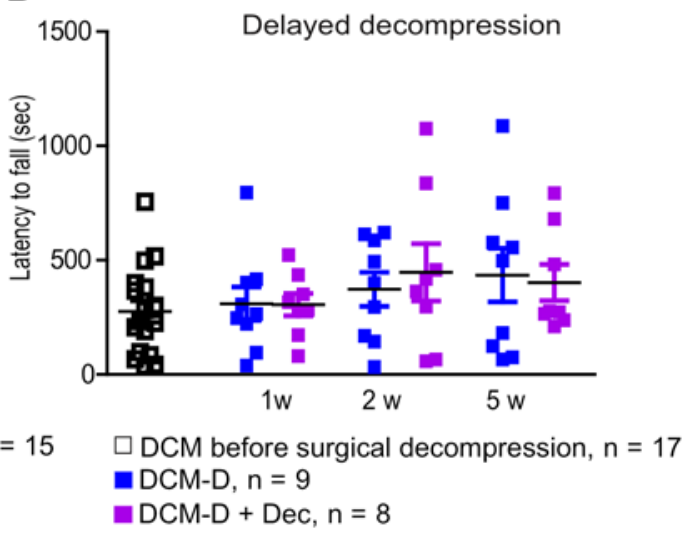

-DCM-D, $\mathrm{n}=9$
C

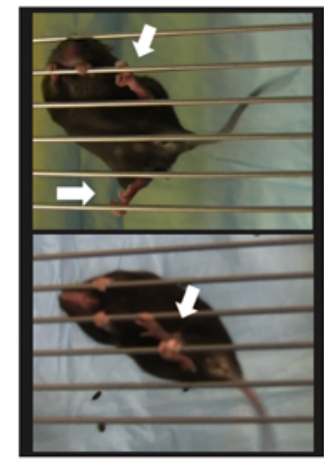

D

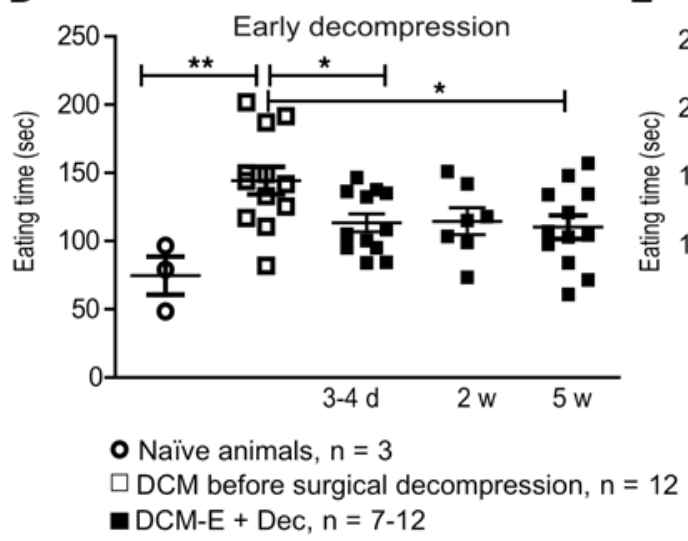

E

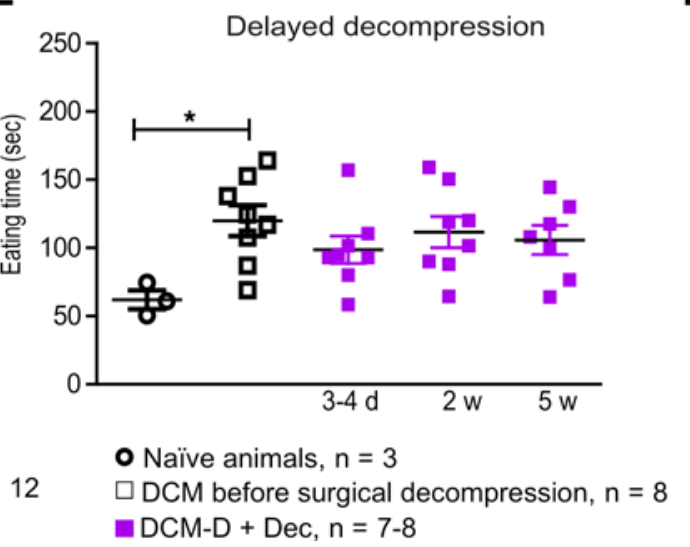

$\mathbf{F}$

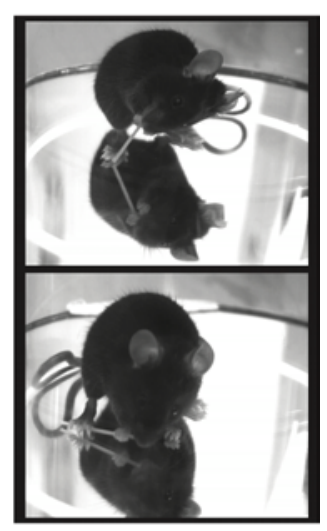

Figure 10. Early surgical decompression improves upper extremity function. (A) Early surgical decompression improves muscle/grip strength as assessed with the wire hang test. The latency to fall from the grid was significantly increased in the DCM-E + Dec group compared with the DCM-E group at the 1-, 2-, and 5-week time points. ${ }^{*} P<0.05$, two-way ANOVA. Open squares at the $-1 w$ (1 week before surgical decompression) denotes all the animals before decompression. (B) No significant changes were observed in the group that underwent delayed surgery at any time point. (C) Representative images of DCM animals performing the wire hang test. The white arrows indicate different ways that decompressed animals hold onto the grid with the 4 limbs. The number of animals used was as follows: DCM-E group before surgical decompression $(n=15) ; \mathrm{DCM}-\mathrm{E}(n=7) ; \mathrm{DCM}-$ $\mathrm{E}+\operatorname{Dec}(n=8)$; DCM-D group before surgical decompression $(n=17)$, DCM-D $(n=9)$; DCM-D + Dec $(n=8)$. The dot plot at each time point represents the mean of 3 measurements per animal. (D) Manual dexterity using the Capellini handling test was measured as the time the animals spent eating a piece of 2.6-cm-long pasta. The DCM-E group before decompression experienced a significant increase in the time spent eating the pasta compared with naive animals. ${ }^{* *} P<0.01$, one-way ANOVA. The DCM-E + Dec group spent less time eating the pasta compared with the DCM-E group, at the 3-day, 4-day, and 5-week time points. ${ }^{*} P<0.05$, one-way ANOVA. (E) Mice in the DCM-D group spent more time eating pasta, compared with naive animals. ${ }^{*} P<0.05$, one-way ANOVA. However, no significant changes were detected at any time point after decompression. (F) Representative images of DCM animals performing the Capellini handling test. Some abnormal eating patterns were observed in DCM animals, such as head tilt and grabbing the pasta with 1 forepaw and hind limb on the floor. Naive animals $(n=3)$; DCM-E group before surgical decompression ( $n=12) ;$ DCM-E + Dec $(n=7-12)$; DCM-D group before surgical decompression $(n=8)$; DCM-D + Dec $(n=7-8)$. The dot plot at each time point represents the mean of 3 measurements per animal. The results are presented as mean \pm SEM. DCM, degenerative cervical myelopathy; Dec, decompression; DCM-E, age-matched early sham decompressed group; DCM-D, age-matched delayed sham decompressed group; w, weeks.

Impact of preoperative duration of symptoms on functional outcomes in human DCM patients. To determine whether duration of symptoms was predictive of outcomes after decompression in DCM patients, we conducted a preclinical experiment comparing functional outcomes between a short and long duration of symptoms ( $<6$ months versus $\geq 6$ months). In a previous analysis of the AOSpine studies, we concluded that patients with a longer duration of symptoms were less likely to achieve a score of 16 or higher on the mJOA. This study, however, did not use a dichotomous cutoff for duration of symptoms nor did it evaluate improvements in function using either change scores or minimum clinically important 
Early surgical decompression mitigates pain response

Early decompression

A

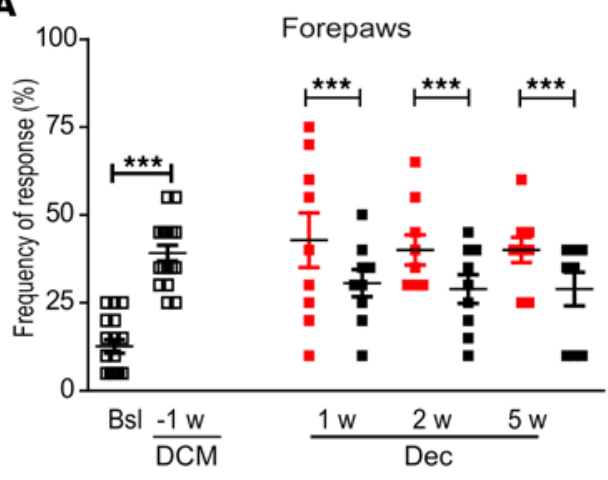

$\square \mathrm{DCM}$ before surgical decompression, $\mathrm{n}=17$

Delayed decompression

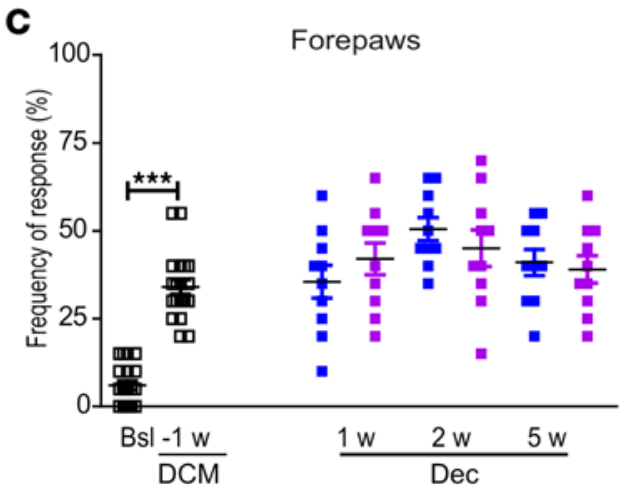

$\square$ DCM before surgical decompression, $n=20$
B

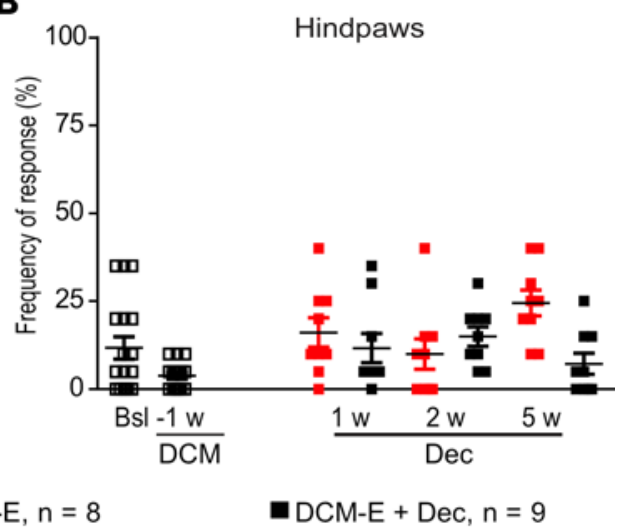

DCM-E, $\mathrm{n}=8$

\section{D}

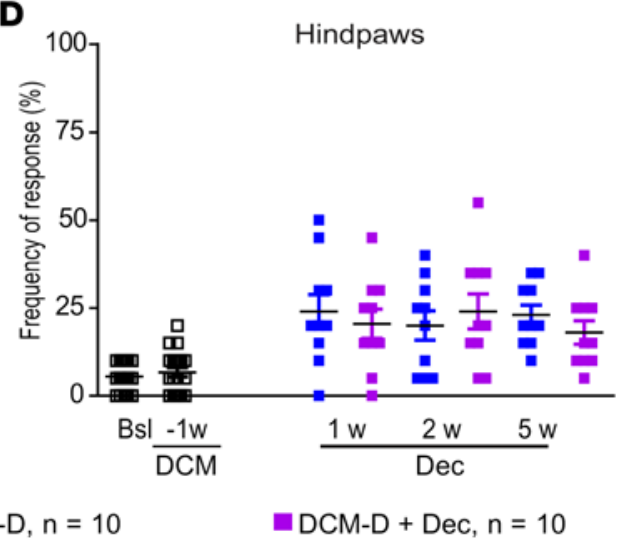

Figure 11. Surgical decompression reduces pain response. Mechanical allodynia was measured in mice in their fore and hind paws using a $0.4-\mathrm{g}$ von Frey hair filament before DCM, during DCM, and after decompression. (A) The early-decompressed group was assessed before DCM (BsI), 1 week before decompression $(-1)$, as well as at 1,2 , and 5 weeks after decompressive surgery. We detected higher responses in the forepaws of animals in the DCM-E group compared with the Bsl time point. ${ }^{* *} P<0.001$, two-tailed $t$ test. Early surgical decompression reduced pain in the forepaws compared with the DCM-E group at all time points studied. ${ }^{* *} P<0.001$, linear mixed model. (B) Pain response in the hind paws of the DCM-E group was not significantly affected at any time point. DCM-E before surgical decompression $(n=17)$; DCM-E $(n=8)$; DCM-E + Dec $(n=9)$. (C) Pain response was significantly increased in the forepaws of DCM-D animals compared with Bsl (*** $P<0.001$, two-tailed $t$ test), without significant changes between the DCM-D and DCM-D + Dec groups. (D) In the hind paws, pain response was not significantly affected at any time point. DCM-D before surgical decompression $(n=20)$; DCM-D $(n=10)$; and DCM-D + Dec $(n=10)$. Each dot plot represents the mean of 10 measurements per paw, per animal. Results are presented as mean \pm SEM. DCM, degenerative cervical myelopathy; Dec, decompression; DCM-E, age-matched early sham decompressed group; DCM-D, age-matched delayed sham decompressed group; w, weeks.

differences (MCIDs). We used an unadjusted model that only controlled for preoperative mJOA and an adjusted model that accounted for other important clinical characteristics. Of the 757 study participants in the CSM AOSpine North America and International studies, 296 (39.10\%) were diagnosed with moderate and $254(33.55 \%)$ with severe disease based on their mJOA score (5) (mJOA 12 to 14 , moderate myelopathy; mJOA 0 to 11 , severe myelopathy). Following imputation of missing data, mJOA scores at 24 months were available for 504 patients (91.63\%) (Table 2). In the unadjusted model (Table 3), patients with a shorter duration of symptoms ( $<6$ months) exhibited a greater improvement on the mJOA score ( $\Delta=4.11 ; 95 \%$ CI, 3.63 to 4.58$)$ than those with a longer duration of symptoms ( $\geq 6$ months) $(\Delta=3.15$; $95 \% \mathrm{CI}, 2.88$ to $3.41 ; P=0.0006)$. This difference remained statistically significant following adjustment for relevant confounders (Table 3 ). In addition, a greater percentage of patients with a shorter duration of symptoms ( $<6$ months) improved by the MCID on the mJOA scale than those with a longer duration of symptoms ( $\geq 6$ months) $(82.2 \%$ versus $67.1 \%, P=0.0016$, Table 3 ). 


\section{Discussion}

In this study, we demonstrated that timing of surgical decompression for DCM impacts the extent and duration of the inflammatory response, the extent of IRI, neurological recovery, and the development of hyperalgesia after decompression. We used a potentially novel clinically relevant mouse model of compression at C5-C6 spinal levels, which represent the most common sites of spinal cord compression in DCM patients (5). This mouse model presents several considerable advantages; similar to our previous rat model, it reproduces the chronic and progressive nature of the human disease and the cinical phenotype, it is MRI compatible, and it allows for surgical decompression (32). However, the mouse model offers the added advantage of using genetically engineered animals in future studies. Previously, we have shown that DCM causes ischemia in the spinal cord, which results in neuronal death and functional deficits in rodents (5). Decompression of the spinal cord may result in IRI, which may explain, in part, suboptimal outcomes in patients treated surgically for DCM as well as neurological complications such as C5 palsy. Nonetheless, surgical decompression is the mainstay treatment in patients with DCM, as it can halt spinal cord compression, minimize further neurological deterioration, and improve functional deficits and quality of life (3).

In this study, surgical decompression was accompanied by an increased production of cytokines acutely within the spinal cord independent of the time of surgical intervention. Delayed surgical decompression resulted in unresolved cytokine production (up to 5 weeks following decompression), sustained astrogliosis, and systemic increase in the ratio of peripheral inflammatory/patrolling blood monocytes. This was accompanied by a lack of neurological improvement. In contrast, early decompression was accompanied by resolution of inflammation and astrogliosis and was associated with neurological recovery in the upper and lower extremities and attenuation of pain in the upper extremities. To our knowledge this study presents the first basic experimental evidence demonstrating the relationship of timing of decompression and the activation of the immune system.

Our findings may contribute to explaining why patients with a longer preoperative duration of symptoms achieve suboptimal surgical outcomes with respect to neurological recovery, functional impairment, and quality of life $(7,42,43)$. We demonstrated that patients with a duration of symptoms greater than or equal to 6 months exhibited smaller functional improvements in functional recovery at 2 years following decompressive surgery than those with a duration of symptoms less than 6 months. Furthermore, a smaller percentage of patients in the longer-duration-of-symptoms group achieved an MCID on the mJOA. The analysis was conducted using a dichotomous variable for duration of symptoms in order to allow for comparison between our preclinical findings and the actual clinical picture. The concordance between our experimental and clinical findings support the need for novel therapeutic targets that can effectively and safely attenuate the detrimental effects associated with postischemia inflammation. Several studies have evaluated important predictors of complications following surgery for DCM. A recent review of the literature indicated that low-level evidence suggests that duration of symptoms is not associated with perioperative complications (44). An analysis of the AOSpine CSM-International dataset confirmed these findings and demonstrated a nonsignificant relationship between complications and preoperative myelopathy duration. However, there is significant heterogeneity in the reporting of complications across centers as well as substantial variation in rates across studies. Prior to further exploration of important predictors of complications, there must be standardization of definitions and the implementation of a classification system.

Clinical studies have reported that almost $9.3 \%$ of patients can deteriorate following a perfect surgical decompression due to the development of IRI within the first 24 hours following decompression (5). Although the manifestation of IRI is complex, there is increasing evidence suggesting that the immune system plays an important role through the initiation of the inflammatory cascade (45). Although in experimental models it has been shown that the effects of inflammation vary, there is significant evidence to suggest that inflammation after IRI correlates with negative outcomes $(46,47)$. In our study, we observed a substantial production of inflammatory cytokines 24 hours after surgical decompression, indicating that there is significant activation of the immune system in a decompressed spinal cord. This finding is in line with previous studies that found increased levels of cytokines following lumbar decompression (48), as well as in spinal and cerebral ischemia $(11,14,15,49)$. These cytokines promote the recruitment of inflammatory cells in the CNS and stimulate the production of matrix metalloproteinases, ROS, and cytokine-associated signaling processes that potentiate a secondary injury phase $(14,46,49,50)$. The deleterious role of inflammation following IRI is further supported by studies demonstrating that attenuation of inflammation can enhance neural repair and functional outcomes $(15,16,51,52)$. Of special interest is the 24-hour time window after decompression, where we have previously 
shown the presence of IRI (5). This time point offers the potential of giving perioperative complementary treatment to surgical decompression that can enhance their benefits, reducing postischemic inflammation. This is the case observed in other CNS IRI models, where pharmacological inhibition of postischemic inflammation helps to reduce lesion size and attenuate ROS production (14-16). These findings support the notion that activation of the immune system coincides with post-IRI decline.

Events of unresolved astrogliosis and inflammation have been reported to be present for months after cerebral ischemia or spinal cord injury (SCI) and correlate with extensive neuronal damage (53-55). We observed similar findings after delayed surgical decompression, where astrogliosis, increased expression of Galectin-3, and secretion of cytokines were elevated in the spinal cord, accompanied by a peripheral increase in the proportion of inflammatory/patrolling monocytes in the blood at 5 weeks after surgery. The increased expression of Galectin-3 might suggest changes in the activation state of $\mathrm{Iba1}^{+}$cells and astrocytes, similar to what is observed after brain ischemia and injury $(30,56)$. Thus, exacerbating inflammation by promoting the secretion of cytokines through activation of the mitogen protein kinase pathways is a relevant downstream mediator for cytokine production $(30,57,58)$. Additionally, increased GFAP, connexin-43, and Iba1 expression after high cervical SCI have been shown to correlate with the development of hyperalgesia, whereas their inhibition can diminish it (59). Given the adverse neurological recovery and the continued hyperalgesia observed after delayed surgical decompression, our results provide evidence that extended duration of immune system activation after surgery may contribute to worse surgical outcomes. It should be noted that the 2 major differences in the immune response between the early and delayed surgical decompression groups was the duration and magnitude of the activation of the immune system. The group that received delayed surgery experienced lower levels of cytokines in the spinal cord compared with the early-decompressed group, but for a longer period of time. Our results are in agreement with the work of others in the literature, showing that the immune response can decrease with age $(17,60)$. Production of cytokines, such as IL-6, TNF- $\alpha$, and IFN- $\gamma$ can also be reduced in the skin of elderly humans, compared with younger patients after antigen challenge (61). The interpretation of the changes observed for CCL-2 and the ratio of peripheral circulating monocytes between DCM-E and DCM-D have to be taken with precaution, since we have observed that these 2 can decrease with the age of the animals.

Herein, the group that underwent early decompressive surgery experienced a shorter period of inflammation and more improvement in upper and lower limb functions compared with the compressed group. However, it should be noted that one of the major changes in gait function of human and rats with DCM is the decrease in the speed and cadence (62). Thus, with progression of DCM the rodents experience a decrease in speed of locomotion that contributes to the more frequent employment of the alternate pattern in our DCM mouse model. Our results are in agreement with results derived from the AOSpine Multi-Center Studies (4), and the Seoul National University Bundang Hospital, which consistently show a beneficial effect of early decompressive surgery in DCM patients $(63,64)$. However, the AOSpine North America Cervical Myelopathy Study did not address the role of the immune system in neurological recovery. Studies of other IRI conditions in the CNS suggest a strong correlation between IRI, inflammation, and neurological recovery. For example, PI3K inhibition resulted in altered microglia activation, decreased production of inflammatory cytokines, and improved neurological outcomes in a cerebral IRI model (65). However, it is worth noting that activation of the immune system and astrocytes is not always detrimental and should not be entirely abolished. Its activation can also promote regeneration by inducing the clearance of regeneration-limiting cellular debris, releasing neurotrophic factors, and limiting the infiltration of other immune cells $(46,50,66-68)$.

Our study has certain limitations. Firstly, duration of symptoms can be subject to recall bias since it relies on patients' perceptions regarding the development of the first symptoms and it might vary if patients present a longer duration of symptoms (3). Secondly, although the duration of compression did not correlate with the extent of reduction in the spinal cord blood flow/ischemia between DCM-E and DCM-D, it correlated with the compression ratio in our animal model of DCM, which is not always the case in DCM patients, where a subcohort of DCM patients can experience stabilization of the compressed spinal cord for long periods of time (44). This makes it possible that neuronal damage, apoptosis (26, 32), and anatomical plasticity can partially contribute to the lack of recovery observed in the group that had a longer compression in our animal model.

The results presented in this study highlight the IRI-mediated inflammation as a previously unrecognized mechanism in the surgical treatment of DCM patients. While the impact of this mechanism on 
patient recovery is not fully elucidated, the prior investigations of IRI models and the data presented herein suggest a correlation with poor functional outcomes. As such, IRI-mediated inflammation may represent a novel therapeutic target to improve DCM patient recovery after surgical decompression. From a clinical perspective, this study also underlines the importance of early diagnosis and surgery in DCM patients. Postoperative recovery can be highly variable among patients undergoing similar surgeries in part due to their prior surgery compensatory ability that can influence neurological assessments (69). For this reason, future studies will need to focus on dissecting the contribution of the immune response before and after surgery, as an additional variable to determine patients' recovery profile. This will enable a more personalized treatment and potentially reduce the costs related to perioperative management.

\section{Methods}

\section{Clinical outcomes}

Seven hundred and fifty-seven patients were prospectively enrolled in either the AOSpine North America ( $n$ $=278$ ) or International $(n=479)$ study at 26 global sites. Patients were recruited to participate in these studies if they met the following inclusion criteria: (a) aged 18 years or older, (b) presenting with symptomatic DCM with at least one clinical sign of myelopathy, (c) evidence of cervical cord compression on MRI or CT, and (d) no previous cervical spine surgery. Patients were excluded if they had active infection, neoplastic disease, rheumatoid arthritis, ankylosing spondylitis, or concomitant lumbar stenosis. At their respective sites, all patients received surgical decompression of the cervical spine. The attending surgeon decided what approach to use, the number of levels to operate on, and whether or not to use fusion or instrumentation. Extensive data were collected at baseline, including basic demographic information, symptomatology, comorbidities, and surgical strategy. Each patient was evaluated preoperatively and at 6, 12, and 24 months postoperatively using a variety of assessment tools, including the mJOA score, the Neck Disability Index, the Nurick classification, the 30-meter walking test, and the Short-Form-36. For this analysis, the mJOA was used as the primary outcome measure; this 18-point scale separately addresses motor dysfunction of the upper and lower extremities, sensory dysfunction of the upper extremities, and micturition (70). The MCID of the mJOA ranges from 1.11 to 1.36 and varies based on myelopathy severity: 1 point for mild $(\mathrm{mJOA} \geq 15), 2$ points for moderate $(\mathrm{mJOA}=12-14)$, and 3 points for severe $(\mathrm{mJOA}<12)$ patients $(71)$.

A mixed-model analytic approach was used to determine the impact of duration of symptoms on postoperative improvements in clinical status. Our analysis focused on patients with moderate (mJOA $=12-14$, $n=296)$ and severe (mJOA $\leq 12, n=254)$ myelopathy (4). Of these 550 patients, $399(72.54 \%)$ attended their 24-month followup visit and were evaluated clinically. A last-value-carried-forward imputation procedure was used to account for missing data; following imputation, mJOA scores at 24 months were available for 504 (91.63\%) patients. The dependent variable was change in mJOA from baseline to 24 postoperative months and the independent variable was duration of symptoms dichotomized at 6 months $(<6$ or $\geq 6)$. In an electronic survey, members of AOSpine International agreed that either a cutoff of 6 or 12 months was the threshold duration of symptoms beyond which there is a negative impact on outcome. As a result, a cutoff of 6 months was used to differentiate between a short and long duration of symptoms (72). Two models were constructed: (a) an unadjusted model that only controlled for preoperative mJOA, and (b) an adjusted model that accounted for other important clinical characteristics (preoperative mJOA, smoking status, age, impaired gait, and psychiatric comorbidities) (42). A secondary analysis was conducted to evaluate differences in the percentage of patients achieving the MCID on the mJOA between duration of symptoms groups ( $<6$ or $\geq 6$ months).

\section{Animals and experimental groups}

We used 148 adult C57BL/ 6 female mice ( 8 weeks old) purchased from The Jackson Laboratory. Animals were divided into 4 groups: (a) DCM with early sham decompression (DCM-E), (b) DCM with early decompression (DCM-E + Dec), (c) DCM with delayed sham decompression (DCM-D), and (d) DCM with delayed decompression (DCM-D + Dec) (Figure 1). Decompression was performed at 6 (herein referred to as early decompression/DCM-E + Dec) or 12 weeks (herein referred to as delayed decompression/DCM-D + Dec) after compression. All surgical procedures were performed under anesthesia using $2 \%$ isoflurane. Animals were sacrificed at 24 hours and 2 and 5 weeks after surgical decompression. All evaluations were performed by 1 or 2 investigators that were blinded to the treatment groups. 


\section{DCM and surgical decompression}

We used a mouse model of DCM that was adapted from a rat DCM model previously established in our laboratory $(5,32)$. Briefly, a C4-T1 midline incision was performed followed by retraction of the paravertebral muscles and subsequent implantation of an aromatic polyether material underneath C5-C6 laminae. This biopolymer induces an osteoid formation with the lamina due to the precipitation of inorganic salts. Surgical decompression was performed as before with relevant changes $(5,32)$. A microdrill was used to slowly and carefully remove the fused laminae and eliminate the compression between the biopolymer and the laminae. Sham-decompressed animals underwent the same surgical procedure as decompressed mice, except for decompression. Muscles and skin were sutured and the animals were monitored daily for the development of neurological complications after surgery.

\section{T1-weighted MR images}

To quantitatively evaluate the compression ratio in our DCM groups, we used MRI as previously described (32). Briefly, mice were maintained at $1.5 \%$ to $2 \%$ isoflurane, and T1-weighted images were acquired using a 7 Tesla system (Bruker BioSpec 70/30 USR, STTARR Innovation Centre) at 2 selected times points: 1 week before decompressive surgery (DCM-E, 5 weeks after material implantation; DCM-D, 11 weeks after material implantation), and 5 weeks after surgical decompression. The compression ratio was calculated using the Medical Image Processing, Analysis, and Visualization (MIPAV) software (NIH). The formula $1-(2 c / a+b) \times 100$ was used to calculate the compression ratio (32), where $c$ is the anteroposterior canal diameter at the level of maximum compression, and $a$ and $b$ are the anteroposterior canal diameter at the nearest normal level above and below the compression site, respectively.

\section{Flow cytometry}

Whole blood was collected via cardiac puncture before perfusion and transferred to EDTA-coated tubes (BD Biosciences). Red blood cells were lysed for 5 minutes (0.1 mM EDTA, $10 \mathrm{mM} \mathrm{KHCO}, 150 \mathrm{mM}$ $\mathrm{NH}_{4} \mathrm{Cl}$ ) and washed twice with PBS. Cells were first stained with viability dye (Fixable Viability Dye eFluor 780, eBioscience) for 20 minutes, followed by 2 washes with $2 \%$ FBS in PBS. A combination of the following antibodies was used to distinguish inflammatory and patrolling monocyte subpopulations in the blood: Ly6C-Pacific blue (clone HK1.4, BioLegend) and CCR2-PE (clone 475301, R\&D Systems), or their respective isotype controls (from BioLegend and R\&D Systems, respectively) for 30 minutes. Data were acquired using a BD LSR II flow cytometer (BD Biosciences) and analyzed using FlowJo (Tree Star).

\section{ELISA and Western blot analysis}

Animals were transcardially perfused with PBS at selected time points ( 24 hours, 2 and 5 weeks) after surgical decompression. The spinal cord was dissected out $(0.5 \mathrm{~cm}$ rostral and $0.5 \mathrm{~cm}$ caudal from the compression epicenter), and homogenized using radioimmunoprecipitation assay (RIPA) buffer with proteinase inhibitors (Thermo Fisher Scientific), according to the manufacturer's instructions. Total protein levels were determined using a BCA Protein Assay Kit (Thermo Fisher Scientific). The homogenates were split and used for ELISA and Western blot. Levels of cytokines of interest were assessed for the same total protein concentration using a mouse 32-panel ELISA system (Eve Technologies). For Western blot analysis, $30 \mu \mathrm{g}$ of total protein was resolved by $12 \%$ SDS-PAGE and transferred to a PVDF membrane. Membranes were blocked with $5 \%$ nonfat milk/0.01\% Tween 20 in PBS for 1 hour at room temperature (RT) and incubated with anti-Galectin-3 antibody (1:1,000; Abcam, ab53082) overnight at $4^{\circ} \mathrm{C}$. Membranes were washed in PBS $/ 0.01 \%$ Tween 20 and incubated with a secondary antibody, goat anti-rabbit conjugated to horseradish peroxidase (1:2,000; Jackson ImmunoResearch, 111-035-144). Membranes were washed and the signal was visualized using Pierce ECL Western Blotting Substrate (Thermo Fisher Scientific) following the manufacturer's instructions. Membranes were stripped with ReBlot Plus Mild Antibody Stripping Solution $1 \times$ (Millipore), blocked for 1 hour, and incubated with anti- $\beta$-actin antibody (1:5,000; Cell Signaling Technology, 12262) at RT. Following membrane exposure, ImageJ (NIH) was used for densitometric analysis of the data. Galectin-3 signal was normalized to $\beta$-actin signal for each lane.

\section{Regional blood flow measurement}

Animals were maintained on spontaneous ventilation using $1.5 \%-2 \%$ isoflurane followed by an incision in the chest as previously described $(73,74)$. Red fluorescent microspheres, $10-\mu$ m diameter $(2: 5$ dilution, 
Thermo Fisher Scientific) were injected into the mouse heart and allowed to circulate through the body for 1 minute according to the manufacturer's instructions. Next, a constant volume of blood sample (reference sample) was collected from the right atrium using a micro-injector pump (World Precision Instruments) with a constant speed for all samples. A portion of the spinal cord around C5-C6 $(0.3 \mathrm{~cm}$ rostro/caudally from the compression epicenter) was collected for regional blood flow measurements, and normalized by the dry weight of the spinal cord portion. Samples were measured using a fluorescence spectrophotometer (Horiba FluoroLog-3) equipped with FluorEssence software. A calibration curve was run every time samples were measured. A second color of fluorescent microparticles was used as an internal standard for blood and spinal cord samples.

\section{Immunohistochemistry and cell quantification}

Animals were transcardially perfused after sham decompression or surgical decompression with PBS followed by $4 \%$ paraformaldehyde. For immunofluorescence analysis, the spinal cords were isolated and postfixed for 4 hours, and then cryoprotected in 30\% sucrose/PBS for 48 hours. Coronal cryosections $(30 \mu \mathrm{m})$ were blocked for 1 hour at RT in blocking solution (10\% nonfat milk, $1 \%$ BSA, $0.3 \%$ Triton $\mathrm{X}-100$ in PBS), followed by incubation with primary antibody overnight (at $4^{\circ} \mathrm{C}$ ), 3 washes with PBS, and incubation with conjugated secondary antibody and 4',6-diamidino-2-phenylindole (DAPI, 1:200, Sigma-Aldrich) for 1 hour at RT. Microglia/macrophage numbers were manually counted through Z-stack $(30 \mu \mathrm{m})$ using the Iba1 marker (1:300, Wako, 019-19741) at $\times 40$ magnification. An anti-GFAP antibody conjugated to Cy3 (1:300, Sigma-Aldrich, MAB3402C3) was used to assess astrogliosis (28) at $\times 20$ magnification. Microglia and GFAP were quantified as the mean of 5 to 6 cryosections/animal, at an interval of $240 \mu \mathrm{m}$ each around the compression epicenter (between C5-C6 segments). The area of the dorsal horns evaluated for GFAP immunoreactivity was traced using ImageJ software and based on DAPI signal in a constant squared region of $89 \times 89 \mu \mathrm{m}$. Iba $1^{+}$cells were quantified in a constant squared region of $214 \times 214 \mu \mathrm{m}$. The integrated density of binary converted images was calculated for each image using ImageJ. All images were captured using a Nikon Eclipse Ti C2+ inverted confocal microscope with NIS element imaging software.

\section{Neurobehavioral analysis}

Mechanical allodynia. Mice were habituated for 1 hour in individual observation chambers with a wire mesh at floor level.

A $0.4 \mathrm{~g}$ von Frey hair monofilament applied 10 times was used to measure the frequency of withdrawal to each forepaw and hind limb, where each withdrawal was measured as $10 \%$ of response (75).

Mechanical sensitivity was assessed at different time points: before DCM induction (baseline, Bsl), 1 week before (-1) surgical decompression, and 3 times after surgical decompression (at 1, 2, and 5 weeks after surgery).

\section{Grip/muscle strength and manual dexterity}

The forelimb wire hang test was used to evaluate neuromuscular grip strength before and after surgical decompression in DCM animals (39). Briefly, mice were placed on a wire cage top, which was then inverted. The latency to fall for each animal was recorded at 1 week before surgical decompression, and at 1 , 2 , and 5 weeks after decompressive surgery. Manual dexterity was assessed using the Capellini handling test as previously described (40). The time animals spent eating a 2.6- $\mathrm{cm}$ piece of pasta was recorded at selected time points ( 1 week before surgical decompression, and at 3 to 4 days and 2 and 5 weeks after surgical decompression). Naive age-matched animals were used for comparison. For both tests, the results are expressed as the average of 3 sessions.

\section{Automated gait analysis}

The CatWalk XT 10.6 (Noldus) system was used to analyze gait deficits in DCM and decompressed animals as described previously (32). We focused our analysis on the following parameters: stride length, swing speed in upper and lower extremities, as well as the step pattern at 5 weeks after sham decompression and surgical decompression. We considered only runs with a minimum of 3 compliant cycles demonstrating a complete gait pattern, where maximum speed variation within each run was less than $50 \%(35)$, and the average speed between runs was not significantly different (33). 


\section{Statistics}

Prism (GraphPad Software) and SPSS (IBM) were used for statistical analysis of data in the animal model, and SAS Software for the human DCM data. The wire hang test, Capellini handling test, ELISA, and flow cytometry results were analyzed using either one- or two-way ANOVA, followed by post hoc test. Comparisons between DCM-E and DCM-D were analyzed using a 2-tailed $t$ test. Immunohistochemical, regional blood flow, and CatWalk analyses were evaluated using a Mann-Whitney $U$ test for comparison between 2 groups. The von Frey results were analyzed using a linear mixed model. The data for DCM patients were analyzed using either a $\chi^{2}$ test or a mixed-model approach as outlined above. All data are given as the mean $\pm \mathrm{SEM}$ in the graphs. Results were considered significant if they had a $P$ value less than 0.05 : ${ }^{*} P<0.05$, ${ }^{* *} P<0.01$, and ${ }^{* * *} P<0.001$.

\section{Study approval}

All animal protocols were approved by the Animal Use Committee (AUC) of the University Health Network (UHN) in Toronto. For human data, written informed consent was obtained from each participant prior to inclusion in the study.

\section{Author contributions}

MGF and PMV conceived the study and the experimental design. SKK and PMV performed surgical procedures. AU and PMV performed flow cytometry experiments. AML, JW, and PMV performed regional blood flow experiments. SF and PMV performed von Frey tests. WF performed MRI acquisition. PMV performed the neurobehavioral test, immunohistochemistry, and cell quantification. LT performed the analysis of the neurological assessment of the CSM North America and International clinical results. AML and PMV performed statistical analysis. All authors contributed to writing the manuscript.

\section{Acknowledgments}

The authors would like to thank the financial support of the 21st Century Research Grant from the Cervical Spine Research Society. We would like to thank Madeleine O'Higgins for assistance with editing and Paul Bradshaw for his critical review of this manuscript.

Address correspondence to: Michael G. Fehlings, Halbert Chair in Neural Repair and Regeneration, The Toronto Western Hospital, University Health Network, Room 4WW-449, 399 Bathurst Street, Toronto, Ontario M5T 2S8, Canada. Phone: 416.603.5627; E-mail: Michael.Fehlings@uhn.ca.

SF's present address is: Department of Neurology and Behavior, UC Irvine, Irvine, California, USA.

1. Nouri A, Tetreault L, Singh A, Karadimas SK, Fehlings MG. Degenerative cervical myelopathy: epidemiology, genetics, and pathogenesis. Spine. 2015;40(12):E675-E693.

2. Tetreault L, et al. Degenerative cervical myelopathy: a spectrum of related disorders affecting the aging spine. Neurosurgery. 2015;77 Suppl 4:S51-S67.

3. Fehlings MG, et al. A global perspective on the outcomes of surgical decompression in patients with cervical spondylotic myelopathy: results from the prospective multicenter AOSpine International study on 479 patients. Spine. 2015;40(17):1322-1328.

4. Fehlings MG, et al. Efficacy and safety of surgical decompression in patients with cervical spondylotic myelopathy: results of the AOSpine North America prospective multi-center study. J Bone Joint Surg Am. 2013;95(18):1651-1658.

5. Karadimas SK, et al. Riluzole blocks perioperative ischemia-reperfusion injury and enhances postdecompression outcomes in cervical spondylotic myelopathy. Sci Transl Med. 2015;7(316):316ra194.

6. Fehlings MG, et al. Perioperative and delayed complications associated with the surgical treatment of cervical spondylotic myelopathy based on 302 patients from the AOSpine North America Cervical Spondylotic Myelopathy Study. J Neurosurg Spine. 2012;16(5):425-432.

7. Tetreault L, Kopjar B, Côté P, Arnold P, Fehlings MG. A clinical prediction rule for functional outcomes in patients undergoing surgery for degenerative cervical myelopathy: analysis of an international prospective multicenter data set of 757 subjects. J Bone Joint Surg Am. 2015;97(24):2038-2046.

8. Gökce EC, et al. Neuroprotective effects of thymoquinone against spinal cord ischemia-reperfusion injury by attenuation of inflammation, oxidative stress, and apoptosis. J Neurosurg Spine. 2016;24(6):949-959.

9. Dimitrijevic OB, Stamatovic SM, Keep RF, Andjelkovic AV. Absence of the chemokine receptor CCR2 protects against cerebral ischemia/reperfusion injury in mice. Stroke. 2007;38(4):1345-1353.

10. Chen Y, et al. Overexpression of monocyte chemoattractant protein 1 in the brain exacerbates ischemic brain injury and is associated with recruitment of inflammatory cells. J Cereb Blood Flow Metab. 2003;23(6):748-755.

11. Smith PD, et al. The evolution of chemokine release supports a bimodal mechanism of spinal cord ischemia and reperfusion 
injury. Circulation. 2012;126(11 Suppl 1):S110-S117.

12. Strecker JK, Minnerup J, Gess B, Ringelstein EB, Schäbitz WR, Schilling M. Monocyte chemoattractant protein-1-deficiency impairs the expression of IL-6, IL-1 $\beta$ and G-CSF after transient focal ischemia in mice. PLoS One. 2011;6(10):e25863.

13. Ormstad H, Aass HC, Lund-Sørensen N, Amthor KF, Sandvik L. Serum levels of cytokines and C-reactive protein in acute ischemic stroke patients, and their relationship to stroke lateralization, type, and infarct volume. J Neurol. 2011;258(4):677-685.

14. Kim JY, Kawabori M, Yenari MA. Innate inflammatory responses in stroke: mechanisms and potential therapeutic targets. Curr Med Chem. 2014;21(18):2076-2097.

15. Zhang N, Komine-Kobayashi M, Tanaka R, Liu M, Mizuno Y, Urabe T. Edaravone reduces early accumulation of oxidative products and sequential inflammatory responses after transient focal ischemia in mice brain. Stroke. 2005;36(10):2220-2225.

16. Li XQ, Wang J, Fang B, Tan WF, Ma H. Intrathecal antagonism of microglial TLR4 reduces inflammatory damage to bloodspinal cord barrier following ischemia/reperfusion injury in rats. Mol Brain. 2014;7:28.

17. Montecino-Rodriguez E, Berent-Maoz B, Dorshkind K. Causes, consequences, and reversal of immune system aging. J Clin Invest. 2013;123(3):958-965.

18. Kalogeris T, Baines CP, Krenz M, Korthuis RJ. Cell biology of ischemia/reperfusion injury. Int Rev Cell Mol Biol. 2012;298:229-317.

19. Linehan E, Fitzgerald DC. Ageing and the immune system: focus on macrophages. Eur J Microbiol Immunol (Bp). 2015;5(1):14-24

20. Gaudillière B, et al. Clinical recovery from surgery correlates with single-cell immune signatures. Sci Transl Med. 2014;6(255):255ra131.

21. Kaito M, et al. Relevance of distinct monocyte subsets to clinical course of ischemic stroke patients. PLoS One. 2013;8(8):e69409.

22. Urra X, et al. Monocyte subtypes predict clinical course and prognosis in human stroke. J Cereb Blood Flow Metab. 2009;29(5):994-1002.

23. Shichita T, Ito M, Yoshimura A. Post-ischemic inflammation regulates neural damage and protection. Front Cell Neurosci. 2014;8:319

24. Morrison HW, Filosa JA. A quantitative spatiotemporal analysis of microglia morphology during ischemic stroke and reperfusion. J Neuroinflammation. 2013;10:4

25. Barreto G, White RE, Ouyang Y, Xu L, Giffard RG. Astrocytes: targets for neuroprotection in stroke. Cent Nerv Syst Agents Med Chem. 2011;11(2):164-173.

26. Yu WR, Liu T, Kiehl TR, Fehlings MG. Human neuropathological and animal model evidence supporting a role for Fas-mediated apoptosis and inflammation in cervical spondylotic myelopathy. Brain. 2011;134(Pt 5):1277-1292.

27. Hirai T, et al. The prevalence and phenotype of activated microglia/macrophages within the spinal cord of the hyperostotic mouse (twy/twy) changes in response to chronic progressive spinal cord compression: implications for human cervical compressive myelopathy. PLoS One. 2013;8(5):e64528.

28. Moon ES, Karadimas SK, Yu WR, Austin JW, Fehlings MG. Riluzole attenuates neuropathic pain and enhances functional recovery in a rodent model of cervical spondylotic myelopathy. Neurobiol Dis. 2014;62:394-406.

29. Lalancette-Hébert M, et al. Galectin-3 is required for resident microglia activation and proliferation in response to ischemic injury. J Neurosci. 2012;32(30):10383-10395.

30. Sirko S, et al. Astrocyte reactivity after brain injury-: the role of galectins 1 and 3. Glia. 2015;63(12):2340-2361.

31. Malone A, Meldrum D, Bolger C. Gait impairment in cervical spondylotic myelopathy: comparison with age- and gendermatched healthy controls. Eur Spine J. 2012;21(12):2456-2466.

32. Karadimas SK, et al. A novel experimental model of cervical spondylotic myelopathy (CSM) to facilitate translational research. Neurobiol Dis. 2013;54:43-58.

33. Machado AS, Darmohray DM, Fayad J, Marques HG, Carey MR. A quantitative framework for whole-body coordination reveals specific deficits in freely walking ataxic mice. Elife. 2015;e07892.

34. Neumann M, et al. Assessing gait impairment following experimental traumatic brain injury in mice. J Neurosci Methods. 2009;176(1):34-44.

35. Batka RJ, Brown TJ, Mcmillan KP, Meadows RM, Jones KJ, Haulcomb MM. The need for speed in rodent locomotion analyses. Anat Rec (Hoboken). 2014;297(10):1839-1864.

36. Koopmans GC, Deumens R, Honig WM, Hamers FP, Steinbusch HW, Joosten EA. The assessment of locomotor function in spinal cord injured rats: the importance of objective analysis of coordination. J Neurotrauma. 2005;22(2):214-225.

37. Bellardita C, Kiehn O. Phenotypic characterization of speed-associated gait changes in mice reveals modular organization of locomotor networks. Curr Biol. 2015;25(11):1426-1436.

38. Amenta PS, Ghobrial GM, Krespan K, Nguyen P, Ali M, Harrop JS. Cervical spondylotic myelopathy in the young adult: a review of the literature and clinical diagnostic criteria in an uncommon demographic. Clin Neurol Neurosurg. 2014;120:68-72.

39. Tan DP, Liu QY, Koshiya N, Gu H, Alkon D. Enhancement of long-term memory retention and short-term synaptic plasticity in cbl-b null mice. Proc Natl Acad Sci U S A. 2006;103(13):5125-5130.

40. Tennant KA, et al. The vermicelli and capellini handling tests: simple quantitative measures of dexterous forepaw function in rats and mice. JVis Exp. 2010;(41):2076.

41. Kalsi-Ryan S, Karadimas SK, Fehlings MG. Cervical spondylotic myelopathy: the clinical phenomenon and the current pathobiology of an increasingly prevalent and devastating disorder. Neuroscientist. 2013;19(4):409-421.

42. Tetreault LA, et al. A clinical prediction model to determine outcomes in patients with cervical spondylotic myelopathy undergoing surgical treatment: data from the prospective, multi-center AOSpine North America study. J Bone Joint Surg Am. 2013;95(18):1659-1666.

43. Tetreault L, et al. Clinical and Surgical Predictors of Complications Following Surgery for the Treatment of Cervical Spondylotic Myelopathy: Results From the Multicenter, Prospective AOSpine International Study of 479 Patients. Neurosurgery. 2016;79(1):33-44.

44. Tetreault L, Ibrahim A, Côté P, Singh A, Fehlings MG. A systematic review of clinical and surgical predictors of complications following surgery for degenerative cervical myelopathy. J Neurosurg Spine. 2016;24(1):77-99.

45. Hausenloy DJ, Yellon DM. Ischaemic conditioning and reperfusion injury. Nat Rev Cardiol. 2016;13(4):193-209. 
46. Iadecola C, Anrather J. The immunology of stroke: from mechanisms to translation. Nat Med. 2011;17(7):796-808.

47. Murray KN, et al. Systemic inflammation impairs tissue reperfusion through endothelin-dependent mechanisms in cerebral ischemia. Stroke. 2014;45(11):3412-3419.

48. Kim KT, Lee SH, Suk KS, Bae SC. The quantitative analysis of tissue injury markers after mini-open lumbar fusion. Spine. 2006;31(6):712-716

49. Offner H, Subramanian S, Parker SM, Afentoulis ME, Vandenbark AA, Hurn PD. Experimental stroke induces massive, rapid activation of the peripheral immune system. J Cereb Blood Flow Metab. 2006;26(5):654-665.

50. Ren X, et al. Regulatory B cells limit CNS inflammation and neurologic deficits in murine experimental stroke. $J$ Neurosci. 2011;31(23):8556-8563.

51. Li XQ, Lv HW, Tan WF, Fang B, Wang H, Ma H. Role of the TLR4 pathway in blood-spinal cord barrier dysfunction during the bimodal stage after ischemia/reperfusion injury in rats. J Neuroinflammation. 2014;11:62.

52. Wong JK, Chen L, Huang Y, Sehba FA, Friedel RH, Zou H. Attenuation of cerebral ischemic injury in Smad1 deficient mice. PLoS One. 2015;10(8):e0136967.

53. Van Beek J, Chan P, Bernaudin M, Petit E, MacKenzie ET, Fontaine M. Glial responses, clusterin, and complement in permanent focal cerebral ischemia in the mouse. Glia. 2000;31(1):39-50.

54. Byrnes KR, Washington PM, Knoblach SM, Hoffman E, Faden AI. Delayed inflammatory mRNA and protein expression after spinal cord injury. J Neuroinflammation. 2011;8:130.

55. Bethea JR. Spinal cord injury-induced inflammation: a dual-edged sword. Prog Brain Res. 2000;128:33-42.

56. Burguillos MA, et al. Microglia-secreted galectin-3 acts as a Toll-like receptor 4 ligand contributes to microglial activation. Cell Rep. 2015;10(9):1626-1638.

57. Filer A, et al. Galectin 3 induces a distinctive pattern of cytokine and chemokine production in rheumatoid synovial fibroblasts via selective signaling pathways. Arthritis Rheum. 2009;60(6):1604-1614.

58. Yan YP, Lang BT, Vemuganti R, Dempsey RJ. Galectin-3 mediates post-ischemic tissue remodeling. Brain Res. 2009;1288:116-124

59. Lee-Kubli CA, et al. Analysis of the behavioral, cellular and molecular characteristics of pain in severe rodent spinal cord injury. Exp Neurol. 2016;278:91-104.

60. Solana R, Tarazona R, Gayoso I, Lesur O, Dupuis G, Fulop T. Innate immunosenescence: effect of aging on cells and receptors of the innate immune system in humans. Semin Immunol. 2012;24(5):331-341.

61. Agius E, et al. Decreased TNF-alpha synthesis by macrophages restricts cutaneous immunosurveillance by memory CD4 ${ }^{+} \mathrm{T}$ cells during aging. J Exp Med. 2009;206(9):1929-1940.

62. Kuhtz-Buschbeck JP, Jöhnk K, Mäder S, Stolze H, Mehdorn M. Analysis of gait in cervical myelopathy. Gait Posture. 1999;9(3):184-189.

63. Lee SE, Jahng TA, Kim HJ. Surgical outcomes in patients with mild symptoms, but severely compressed spinal cord from cervical ossification of the posterior longitudinal ligament. J Clin Neurosci. 2016;33:163-168.

64. Tetreault L, et al. Predicting the minimum clinically important difference in patients undergoing surgery for the treatment of degenerative cervical myelopathy. Neurosurg Focus. 2016;40(6):E14.

65. Wang P, et al. Class I PI3K inhibitor ZSTK474 mediates a shift in microglial/macrophage phenotype and inhibits inflammatory response in mice with cerebral ischemia/reperfusion injury. J Neuroinflammation. 2016;13(1):192.

66. David S, Kroner A. Repertoire of microglial and macrophage responses after spinal cord injury. Nat Rev Neurosci. 2011;12(7):388-399.

67. Peruzzotti-Jametti L, Donegá M, Giusto E, Mallucci G, Marchetti B, Pluchino S. The role of the immune system in central nervous system plasticity after acute injury. Neuroscience. 2014;283:210-221.

68. Liu W, Tang Y, Feng J. Cross talk between activation of microglia and astrocytes in pathological conditions in the central nervous system. Life Sci. 2011;89(5-6):141-146.

69. Aleksanderek I, McGregor SM, Stevens TK, Goncalves S, Bartha R, Duggal N. Cervical spondylotic myelopathy: metabolite changes in the primary motor cortex after surgery. Radiology. 2017;282(3):817-825.

70. Benzel EC, Lancon J, Kesterson L, Hadden T. Cervical laminectomy and dentate ligament section for cervical spondylotic myelopathy. J Spinal Disord. 1991;4(3):286-295.

71. Tetreault L, Nouri A, Kopjar B, Côté P, Fehlings MG. The minimum clinically important difference of the modified Japanese Orthopaedic Association scale in patients with degenerative cervical myelopathy. Spine. 2015;40(21):1653-1659.

72. Tetreault LA, Nouri A, Singh A, Fawcett M, Fehlings MG. Predictors of outcome in patients with cervical spondylotic myelopathy undergoing surgical treatment: a survey of members from AOSpine International. World Neurosurg. 2014;81(3-4):623-633.

73. Kurokawa R, Murata H, Ogino M, Ueki K, Kim P. Altered blood flow distribution in the rat spinal cord under chronic compression. Spine. 2011;36(13):1006-1009.

74. Serrat MA. Measuring bone blood supply in mice using fluorescent microspheres. Nat Protoc. 2009;4(12):1779-1758

75. Dietrich F, et al. The quinovic acid glycosides purified fraction from Uncaria tomentosa protects against hemorrhagic cystitis induced by cyclophosphamide in mice. PLoS One. 2015;10(7):e0131882.

76. Fehlings MG, Tator $\mathrm{CH}$. An evidence-based review of decompressive surgery in acute spinal cord injury: rationale, indications, and timing based on experimental and clinical studies. J Neurosurg. 1999;91(1 Suppl):1-11. 